ARTICLE

\title{
Dissecting cell-type-specific roles of androgen receptor in prostate homeostasis and regeneration through lineage tracing
}

Qing Xie ${ }^{1}$, Yueli Liu ${ }^{1}$, Tao Cai ${ }^{2}$, Corrigan Horton ${ }^{1}$, Joshua Stefanson ${ }^{1} \&$ Zhu A. Wang ${ }^{1}$

Androgen signals through androgen receptor $(A R)$ to influence prostate development and cancer. How stromal and epithelial AR regulate prostate homeostasis remains unclear. Using genetic lineage tracing, we systematically investigated the role of cell-autonomous $A R$ in different prostate epithelial cell types. Here we show that AR is dispensable for basal cell maintenance, but is cell-autonomously required for the luminal differentiation of rare basal stem cells. In contrast, AR deletion in luminal cells alters cell morphology and induces transient over-proliferation, without affecting androgen-mediated luminal cell survival or regeneration. However, AR is selectively required for the maintenance of daughter cells produced by castration-resistant Nkx3.1-expressing luminal stem cells (CARNs). Notably, Pten loss can override AR-loss effects in both basal and luminal compartments to initiate tumours. Our data reveal distinct cell-type-specific roles of epithelial AR in orchestrating prostate homeostasis, and question the notion that epithelial AR serves as a tumour suppressor in early cancer initiation.

\footnotetext{
${ }^{1}$ Department of Molecular, Cell and Developmental Biology, University of California, Santa Cruz, California 95064, USA. ${ }^{2}$ Sequencing Center, National Institute of Biological Sciences, Beijing 102206, China. Correspondence and requests for materials should be addressed to Z.A.W. (email: zwang36@ucsc.edu).
} 
T he steroid hormone, androgen, plays critical roles in prostate development and cancer progression through its nuclear receptor, androgen receptor $(\mathrm{AR})^{1,2}$. However, the specific functions of AR in those processes remain elusive, and investigations into this question are complicated by the dynamic and heterogeneous expression pattern of AR in different prostate cell types through time. The prostate gland consists of stromal tissues that mainly include interstitial fibroblasts and smooth muscle cells, as well as an epithelium that includes rare neuroendocrine cells and two major cell types, namely, basal cells that express cytokeratin (CK) 5, p63 and low levels of AR, and luminal cells that express CK18, Nkx3.1 and high levels of $\mathrm{AR}^{3}$. Classic tissue recombination experiments showed that stromal AR, but not epithelial AR, is essential for prostate developmental growth and morphogenesis through paracrine signals $^{4-6}$. Later mouse genetic studies using AR conditional knockout in stromal fibroblasts and/or smooth muscle cells also reported reduced prostate size, decreased epithelial proliferation and impaired histology ${ }^{7-10}$; although the tumour-suppressing/ promoting role for stromal AR during prostate cancer progression is still debated ${ }^{11,12}$. In contrast, in the prostate epithelium, AR function has traditionally been thought to primarily regulate the expression of androgen-dependent secretory proteins ${ }^{1,13}$. Recently, several Cre lines were used to ablate $A R$ in the mouse prostate epithelium during postnatal development. While some studies reported a tumour-suppressing role for epithelial AR, others reported varied and sometimes contradictory phenotypes concerning the behaviours of basal and luminal cells ${ }^{14-17}$. To date, the function of $A R$ in the adult prostate epithelium, particularly at the resolution of specific adult epithelial cell types, remains unclear. Acquiring such knowledge will be crucial for our understanding of prostate homeostasis and cancer initiation.

Recent mouse lineage-tracing studies from multiple groups have provided a clearer picture of the cell lineage relationship in the normal prostate epithelium in vivo. Whereas basal cells serve as multipotent stem cells to generate luminal and neuroendocrine cells during prostate development ${ }^{18}$, their stem cell activities become gradually restricted as the prostate organ matures ${ }^{19}$. In adult prostate homeostasis, basal and luminal cells are largely two self-sustained lineages, with very low proliferation in both compartments and basal-to-luminal cell differentiation occurring only occasionally ${ }^{19,20}$. When androgen is deprived through castration, the prostate will regress as the majority of luminal cells undergo cell apoptosis while basal cells are largely unaffected. Re-administration of androgen will lead to luminal cell regeneration and prostate growing back to normal size. Such androgen-mediated prostate regression-regeneration can be repeated for multiple cycles in rodents ${ }^{21}$. Lineage-tracing analyses showed that luminal cell regeneration is primarily due to the proliferation of the remaining luminal cells that survive androgen deprivation ${ }^{20,22}$, although rare basal stem cell (BSC) activities also minimally contribute ${ }^{19}$. Whether cell-autonomous $A R$ in those cell types drives regeneration in response to androgen is unknown. In addition, while all luminal cells in the hormonal-intact prostate express the transcription factor Nkx3.1, in the regressed prostate, Nkx3.1-expressing luminal cells (named CARNs) are rare, and they were shown to behave as a type of luminal stem cell that can produce both luminal and basal cells during regeneration ${ }^{23}$. Since $N k \times 3.1$ is a downstream target gene of $\mathrm{AR}^{24,25}$, the role of $\mathrm{AR}$ in CARNs awaits to be investigated.

Deletion of the tumour suppressor gene Pten in the mouse prostate epithelium has served as a highly relevant model for studying human prostate cancer $^{26}$. Under this oncogenic condition, basal, luminal and CARN cells all can serve as the cell of origin for prostate cancer ${ }^{19,20,23,27}$. Recently, it was shown that epithelial AR in general is not required for the initiation and progression of Pten-null prostate cancer $^{28}$. However, whether basal- and luminal-specific ARs play different roles in tumour formation is unknown. Here, we set out to investigate the role of AR in different prostate epithelial cell lineages in the context of adult prostate homeostasis, androgen-mediated prostate regression-regeneration and tumour initiation. Through cell-type-specific ablation of AR coupled with lineage-tracing analyses, our data demonstrate distinct AR functions in adult basal and luminal cells, and uncover its essential roles in the multipotent capability of rare stem cells in both compartments.

\section{Results}

Heterogeneous expression and dispensability of basal cell AR. AR has been considered absent or expressed at very low levels in adult prostate basal cells, but highly expressed in luminal cells. Our immunofluorescence (IF) staining of adult mouse prostate confirmed strong nuclear AR expression in all luminal cells (Fig. 1a), and interestingly, revealed its expression to be heterogeneous in the basal layer, as strong AR nuclear staining was randomly present in a subset of basal cells (Fig. 1a). For lineage analysis of basal cells, we used the previously characterized inducible basal-specific driver $C K 5-C r e E R^{T 2}$ (refs 19,29). For better visualization and quantitation of basal AR, we tamoxifeninduced CK5-CreER $R^{T 2}$ R26R-CAG-YFP/ + (denoted Bas ${ }^{\mathrm{YFP}}$ ) mice, in which almost all of the basal cells $(98.7 \%$, $n=8,807 / 8,921$, three animals analysed) can be marked by a CAG promoter-driven enhanced $\mathrm{YFP}^{30}$, with $58.7 \%$ of the marked cells positive for AR staining (Fig. 1b,f; Supplementary Table 1).

To test whether AR is functionally significant in adult basal cells, we conditionally deleted the AR gene in them by tamoxifen induction of 8-week old CK5-CreER ${ }^{T 2}$; AR flox/Y $; R 26 R-C A G-Y F P /+$ (denoted Bas ${ }^{\mathrm{YFP}, \mathrm{AR}-}{ }^{-}$) male mice and performed lineage tracing (Fig. 1c). The $A R^{f l o x}$ allele deletes exon 2 upon induction, leading to disruption of the sequence encoding the DNA binding domain and yielding a non-functional transcript harbouring a frame shift and premature stop $\operatorname{codon}^{31,32}$. We found basal AR deletion to be efficient but not fully penetrant, as the percentage of $\mathrm{YFP}^{+}$basal cells that were $\mathrm{AR}^{+}$significantly decreased to $22.2 \%$ in the anterior prostate (AP) lobes 2 weeks after induction (three animals analysed, $P<0.001$ by $t$-test, Fig. 1 d,f; Supplementary Table 1). In the subsequent tracing period of up to 8 months of age, no abnormality of the prostatic tubule structure or morphological changes of either basal or luminal cells were observed (Fig. 1e), and the percentage of $\mathrm{AR}^{+}$basal cells remained stable at the reduced ratio (Fig. 1f; Supplementary Table 1). Furthermore, cleaved Caspase 3 staining revealed no elevation of apoptosis in the epithelium, and both BrdU incorporation assays (Fig. 1c) and Ki67 staining showed that the proliferation rates in $\mathrm{AR}^{+}$and $\mathrm{AR}^{-}$basal cells were similar (Fig. 1g,h; Supplementary Fig. 1; Supplementary Table 1). AR deletion was less efficient in the ventral prostate and dorsal-lateral prostate lobes than in the AP, but the proportions of $\mathrm{AR}^{+}$and $\mathrm{AR}^{-}$basal cells also remained stable throughout the tracing period (Supplementary Fig. 2). Taken together, we conclude that AR expression in adult prostate basal cells is dispensable for their normal homeostasis.

AR is cell-autonomously required for BSC differentiation. Prostate epithelial cells are normally lineage-restricted in the adult organ, with rare basal cells occasionally undergoing luminal differentiation in vivo ${ }^{19,20}$. Consistent with previous reports, we found that, in the AP lobes of 8-month-old Bas ${ }^{\mathrm{YFP}}$ mice that had undergone lineage tracing (Fig. 1c), $1.1 \%$ of $\mathrm{YFP}^{+}$cells were 
a

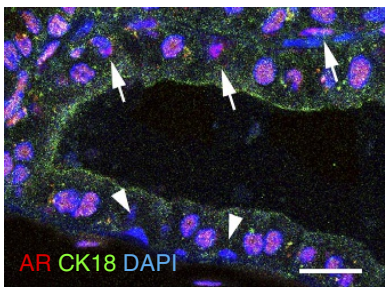

b

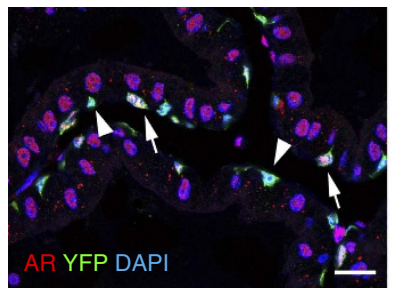

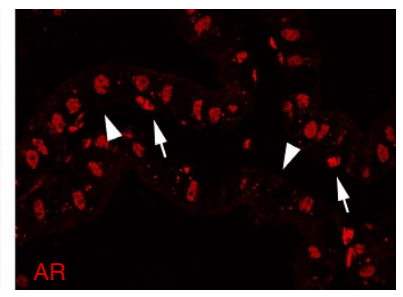

C

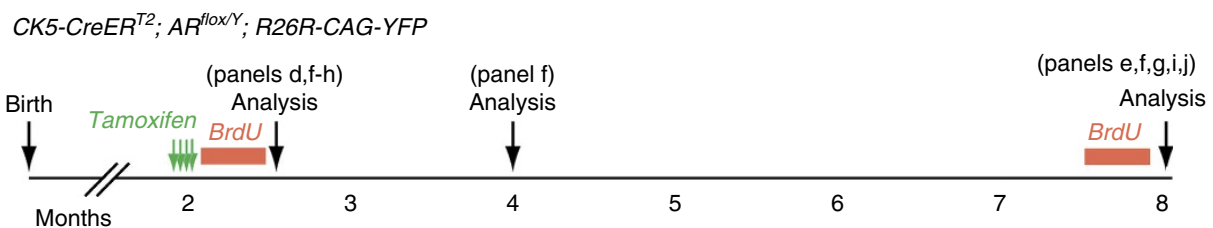

d

e
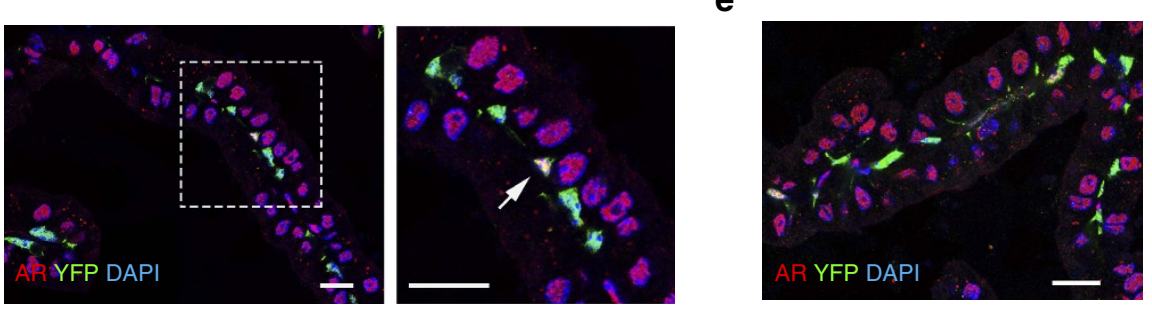

f

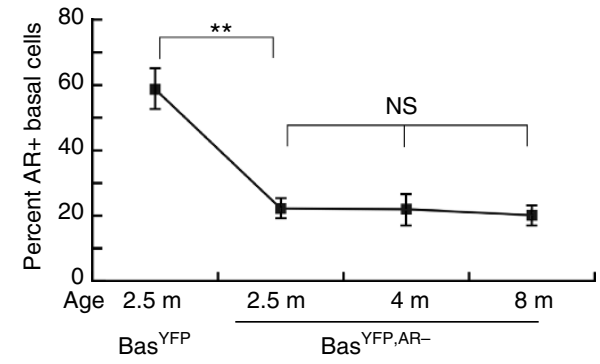

g

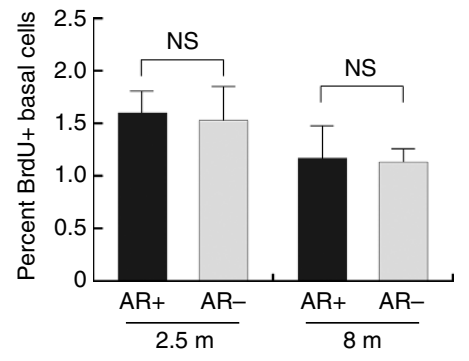

h
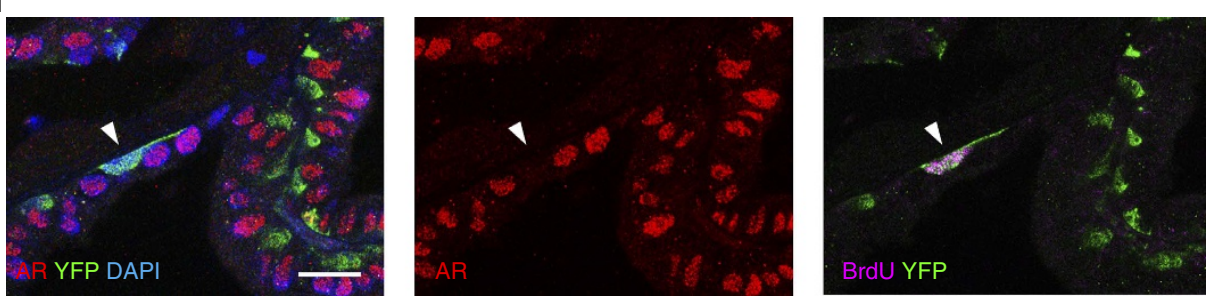

i

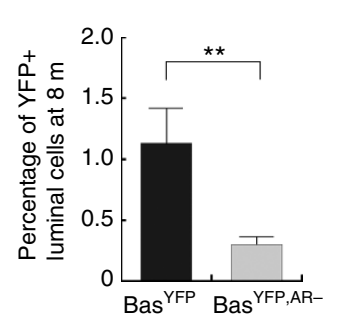

j
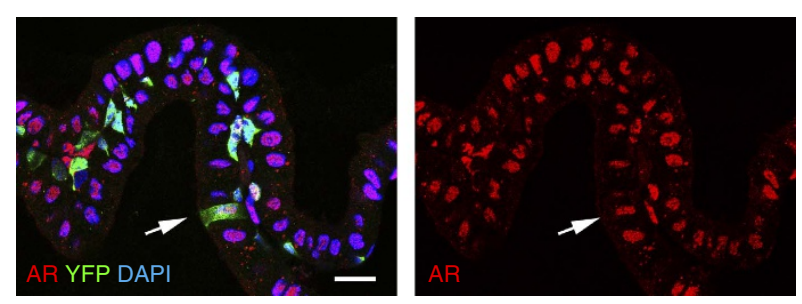

Figure 1 | Lineage analysis of $\mathbf{A} \mathbf{R}^{-}$and $\mathbf{A R}{ }^{+}$basal cells in prostate homeostasis. (a) AR expression in all the luminal cells and the presence of $A R^{+}$ (arrows) and $\mathrm{AR}^{-}$(arrowheads) basal cells. (b) Lineage-marking of basal cells by CAG-YFP is highly penetrant and facilitates quantitation of AR ${ }^{+}$

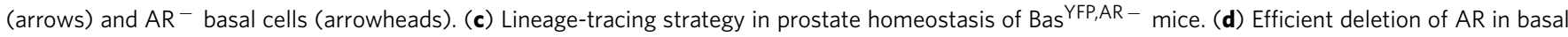
cells at 2 weeks post induction. (e) Normal tissue morphology and absence of AR in the majority of basal cells at 6 months after induction. (f) Percentage of $\mathrm{AR}^{+}$basal cells decreased significantly after AR deletion and remained stable thereafter in homeostasis. ${ }^{\star \star} P<0.001$ by $t$-test. ( $(\mathbf{g}) \mathrm{AR}^{+}$and $\mathrm{AR}^{-}$basal cells have the same proliferation rates in BrdU incorporation assays of Bas YFP,AR - mice during homeostasis. NS by t-test. (h) Representative image of BrdU staining analysed at $2.5 \mathrm{~m}$. Arrowhead points to an $\mathrm{AR}^{-}$basal cell that is $\mathrm{BrdU}^{+}$. (i) Percentage of $\mathrm{YFP}^{+}$luminal cells decreased significantly in Bas ${ }^{Y F P, A R}-$ mice at 6 months post induction compared to Bas ${ }^{Y F P}$ mice. ${ }^{\star \star} P<0.0001$ by $\chi^{2}$ test. (j) The rare $\mathrm{YFP}^{+}$luminal cells were always AR ${ }^{+}$(arrow). Scale bars correspond to $20 \mu \mathrm{m}$. Error bars correspond to one s.d. 
luminal ( $n=59 / 5,177$, three animals analysed; Fig. 1i; Supplementary Table 1). In contrast, this basal-to-luminal differentiation ratio significantly decreased to $0.31 \%$ ( $n=17 / 5,545$, three animals analysed, $P<0.0001$ by $\chi^{2}$ test $)$ in lineage-traced 8-month-old Bas $\mathrm{YFP}, \mathrm{AR}-$ mice (Fig. 1i; Supplementary Table 1). Importantly, all of the rare $\mathrm{YFP}^{+}$ luminal cells were $\mathrm{AR}^{+}$(Fig. 1j; Supplementary Fig. 2b, right panel), suggesting they were derived from basal cells that escaped AR deletion. In fact, we never observed even a single AR-negative $\mathrm{YFP}^{+}$luminal cell from Bas ${ }^{\mathrm{YFP}}$ or Bas ${ }^{\mathrm{YFP}, \mathrm{AR}-}$ mice $(n=0 / 76$, six animals analysed, Supplementary Table 1). These data demonstrate that $\mathrm{AR}$ is cell-autonomously required for the bipotentiality of rare adult BSCs in organ homeostasis.

We next studied the behaviours of $\mathrm{AR}^{-}$basal cells in the context of androgen-mediated prostate regression-regeneration. $\mathrm{Bas}{ }^{\mathrm{YFP}, \mathrm{AR}-}$ mice were induced at 8 -weeks of age and then castrated 2 weeks later (Fig. 2a). We found that $21.5 \%$ of the basal cells in the regressed prostate remained $\mathrm{AR}^{+}$(Fig. 2b,d; Supplementary Table 2), a ratio similar to that found before castration. During subsequent serial regression-regeneration of up to three rounds, the percentage of $\mathrm{AR}^{+}$basal cells remained constantly around 20\% (Fig. 2c,d; Supplementary Table 2). Similar results were obtained in the ventral prostate and dorsal-lateral prostate lobes (Supplementary Fig. 3). Therefore, androgen levels do not appear to affect the relative balance between $\mathrm{AR}^{+}$and $\mathrm{AR}^{-}$basal cells. In lineage-traced Bas ${ }^{Y F P}$ mice that had undergone three rounds of prostate regression-regeneration, we found that $0.89 \%$ of the $\mathrm{YFP}^{+}$cells were luminal $(n=71 / 7,990$, three animals analysed, Fig. 2e; Supplementary Table 2), consistent with the previous finding that rare BSCs can give rise to a small proportion of luminal cells during serial regeneration ${ }^{19}$. In contrast, the luminal YFP ${ }^{+}$ ratio significantly decreased to $0.27 \%$ in $\mathrm{Bas}{ }^{\mathrm{YFP}, \mathrm{AR}-}$ mice $\left(n=23 / 8,585\right.$, three animals analysed, $P<0.0001$ by $\chi^{2}$ test, Fig. 2e; Supplementary Table 2). Again, all of the rare YFP ${ }^{+}$ luminal cells were $\mathrm{AR}^{+}$(Fig. 2f; Supplementary Fig. 3b, right panel), and AR-negative YFP ${ }^{+}$luminal cells were never found in $\mathrm{Bas}^{\mathrm{YFP}}$ or Bas ${ }^{\mathrm{YFP}, \mathrm{AR}-}$ mice $(n=0 / 102,12$ animals analysed, Supplementary Table 2). Therefore, cell-autonomous AR is also required for BSCs to differentiate into luminal cells in prostate regeneration.

To corroborate these lineage-tracing findings, we investigated the role of AR in BSCs using the recently developed organoid technique ${ }^{33-35}$. $\mathrm{YFP}^{+}$basal cells were flow-sorted from tamoxifen-induced Bas ${ }^{\mathrm{YFP}}$ and Bas ${ }^{\mathrm{YFP}, \mathrm{AR}}$ - mice, respectively, and 10,000 cells were seeded per well and cultured using a

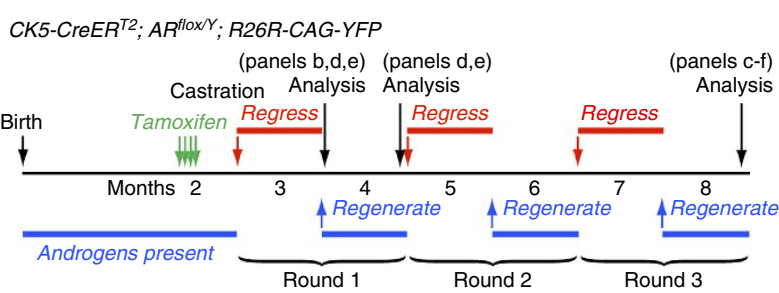

d

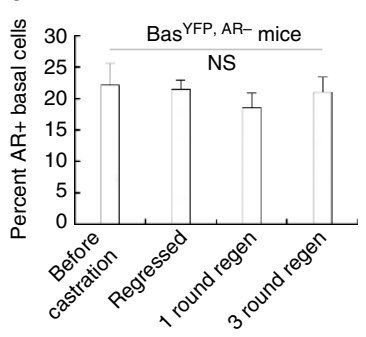

g
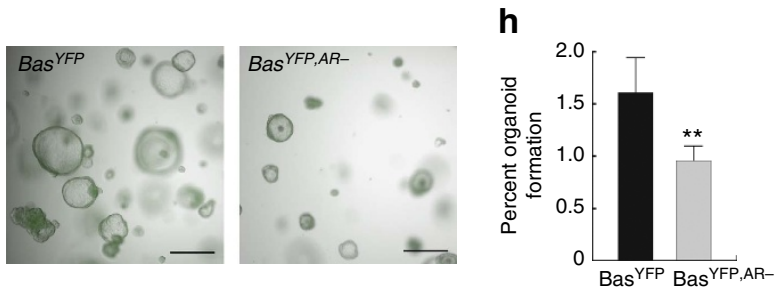
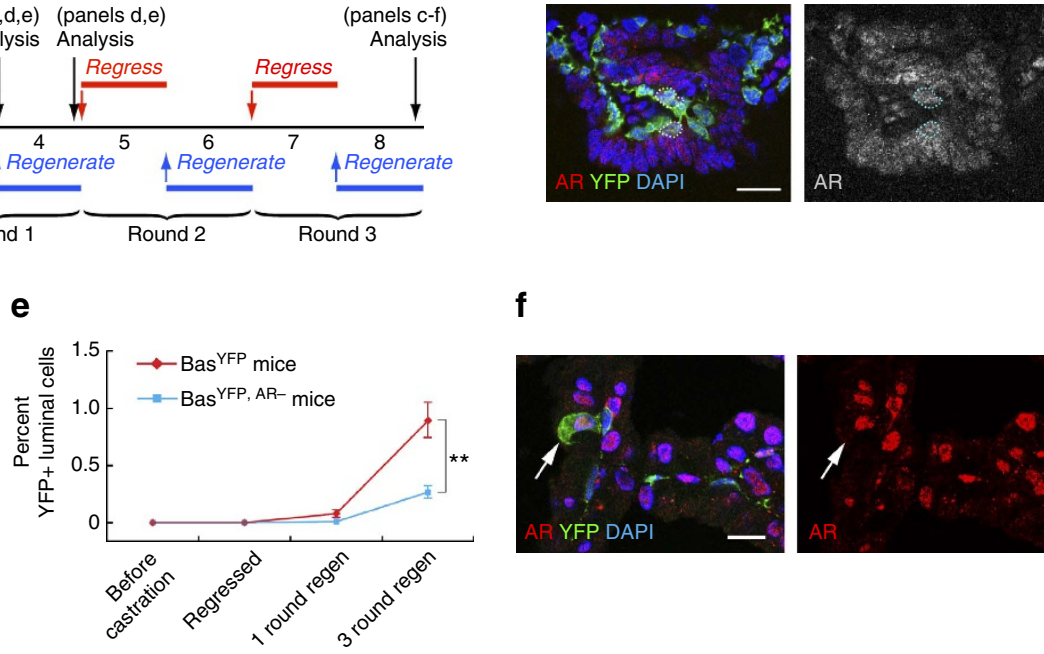
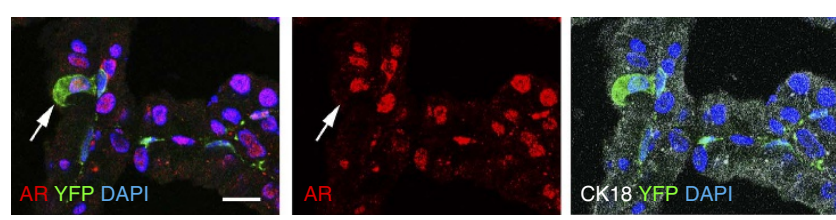

C

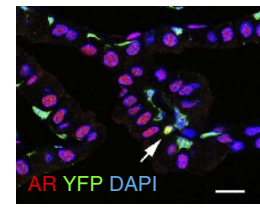

$\mathbf{f}$

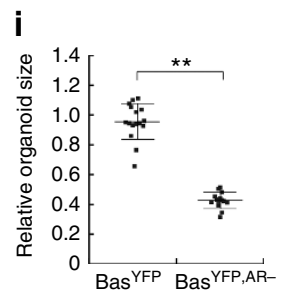

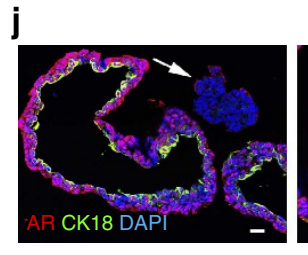

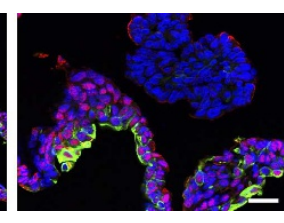

Figure 2 | Cell-autonomous requirement of AR in BSCs in vivo and in organoid culture. (a) Lineage-tracing strategy in serial regression/regeneration

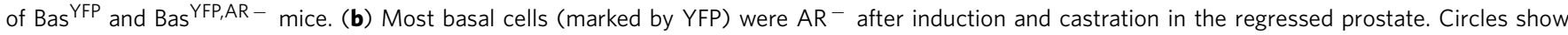
$\mathrm{AR}^{+}$basal cells that escaped AR deletion. (c) Normal cell morphology and few YFP ${ }^{+}$luminal cells after three rounds of regression-regeneration. Most basal cells remained $A R^{-}$. Arrow points to an $A R^{+}$basal cells. (d) Percentage of $A R^{+}$basal cells stayed constant in Bas ${ }^{Y F P, A R}-$ mice during serial regression-regeneration. NS by $t$-test. (e) Percentage of luminal cells among total YFP ${ }^{+}$cells gradually increased in Bas ${ }^{Y F P}$ mice during serial regeneration, but was significantly reduced in Bas $Y F P, A R-$ mice. ${ }^{\star \star} P<0.0001$ by $\chi^{2}$ test. (f) Rare YFP + luminal cells after 3 rounds of regression-regeneration in Bas YFP,AR - mice were always AR ${ }^{+}$(arrow). (g) White field and YFP overlay images showing morphology and abundance of organoids derived from basal cells of Bas ${ }^{Y F P}$ and Bas ${ }^{Y F P}, A R-$ mice. (h) Bar graph comparing organoid formation efficiencies from 16 wells of seeded Bas ${ }^{\text {YFP }}$ cells and 14 wells of seeded Bas ${ }^{Y F P, A R}-$ cells. ${ }^{\star \star} P<0.001$ by $t$-test. (i) Comparison of average organoid size (normalized) per well for 16 wells of seeded Bas ${ }^{Y F P}$ cells and 14 wells of seeded Bas ${ }^{Y F P, A R}$ - cells. ${ }^{\star \star} P<0.001$ by $t$-test. (j) IF section staining showing lack of hollow lumen and little basal-to-luminal differentiation in an AR ${ }^{-}$ basal organoid (arrow and zoom-in on the right) compared to adjacent wild-type basal-derived organoids. Scale bars in $\mathbf{b}, \mathbf{c}, \mathbf{f}, \mathbf{j}$ correspond to $20 \mu \mathrm{m}$, and in $\mathbf{g}$ to $0.5 \mathrm{~mm}$. Error bars correspond to one s.d. 
previous protocol ${ }^{36}$. Basal cells isolated from Bas ${ }^{\mathrm{YFP}}$ mice yielded a significantly greater number of organoids than those from Bas YFP,AR - mice (Fig. 2 g,h, $P<0.001$ by $t$-test), and they also had significantly larger average sizes and more branching (Fig. 2g,i, $P<0.001$ by $t$-test). Since the seeded Bas ${ }^{\text {YFP,AR }-}$ cells were a mixed population of wild type and AR-deleted basal cells, IF staining of individual organoids showed that, compared with wild-type controls, organoids grown from $\mathrm{AR}^{-}$basal cells lacked the hollow lumen and had little basal-to-luminal cell differentiation as revealed by CK18 staining (Fig. 2j). Therefore, these in vitro data also support our conclusions drawn from in vivo lineage tracing experiments.

$A R^{-}$luminal cells expand transiently with altered morphology. Since AR is strongly expressed in the nuclei of all adult luminal cells, we next investigated the effects of luminal AR loss-offunction using the luminal-specific driver $N k \times 3.1^{\text {CreERT2/+ }}$ (ref. 23). Nkx3.1 CreERT2/+ $^{2} A R^{\text {flox/Y }} ;$ R26R-YFP/+ (denoted Lum $^{\text {YFP,AR }-)}$ ) mice were tamoxifen-induced at 8 weeks of age and analysed through adult homeostasis (Fig. 3a). IF staining revealed that YFP fluorescence can reliably indicate AR deletion, since almost all $\mathrm{YFP}^{+}$cells $(98.7 \%, n=1,698 / 1,720$, four animals analysed) were also $\mathrm{AR}^{-}$, while $84.8 \%$ of $\mathrm{AR}^{-}$cells ( $n=1,698 / 2,002$, four animals analysed) were also $\mathrm{YFP}^{+}$ (Fig. 3b, Supplementary Fig. 4a). To further validate the cell type specificity of AR expression, we utilized an established flowsorting protocol ${ }^{37,38}$ to isolate basal cells ( $\left.\mathrm{Lin}^{-} \mathrm{CD}_{4} 9 \mathrm{f}^{\text {hi }} \mathrm{Sca}-1^{+}\right)$,

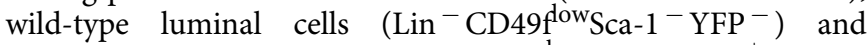
AR-deleted luminal cells ( $\mathrm{Lin}^{-} \mathrm{CD} 49 \mathrm{f}^{\text {low }} \mathrm{Sca}-1^{-} \mathrm{YFP}^{+}$) from

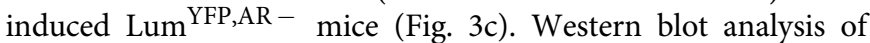
sorted cells confirmed that AR expression is higher in wild-type luminal cells than basal cells, but is absent in AR-deleted luminal cells (Fig. 3d).

During the tracing period, we did not observe any luminal cell sloughing into the lumen or elevation of luminal cell apoptosis by cleaved Caspase 3 staining, suggesting $\mathrm{AR}$ is not cellautonomously required for luminal cell survival. Strikingly, within 2 weeks after induction the $\mathrm{AR}^{-}$luminal cells appeared as condensed cell clusters that were morphologically distinguished from wild-type luminal cells, as these cells were more compact and usually in stacked layers (Fig. 3e). BrdU incorporation assays (Fig. 3a) and Ki67 staining both showed that $\mathrm{AR}^{-}$luminal cells experienced a burst of fast proliferation during the first 1-2 weeks post induction (Fig. 3f,g; Supplementary Fig. 4b,f; Supplementary Table 3), but after this brief period, their proliferation rates decreased back to normal levels that were similar to wild-type luminal cells (Fig. 3f,h; Supplementary Fig. 4c,f; Supplementary Table 3). Such initial over-proliferation explains the origin of the observed condensed $\mathrm{AR}^{-}$luminal cell clusters, and its transient nature agrees with our lineage-tracing data, which showed that from 2 weeks post induction onwards, the ratio of $\mathrm{AR}^{-}$or $\mathrm{YFP}^{+}$luminal cells did not increase through time (Fig. 3i; Supplementary Table 3). Notably, no difference in overall basal cell proliferation was observed during the tracing period (Supplementary Fig. 5).

To further characterize the $\mathrm{AR}^{-}$luminal cells morphologically, we performed staining with different cell markers. $\mathrm{AR}^{-}$ luminal cells retained some luminal features, as they showed enhanced CK18 expression compared with wild-type ones, and no detectable CK5 expression (Fig. 3j). Interestingly, both IF and IHC staining using two different antibodies showed that expression of another basal marker p63 was enhanced in the cytoplasm of $\mathrm{AR}^{-}$luminal cells (Fig. 3k), suggesting they may resemble intermediate cells. Furthermore, Nkx3.1 expression in these cells was abolished (Supplementary Fig. 4d), suggesting it is a downstream target gene of cell-autonomous $\mathrm{AR}^{24,25}$. $\alpha \mathrm{PKC}$, a polarity marker that is normally expressed on the apical side of luminal cells, and E-Cadherin, which is normally expressed on the lateral sides $^{39}$, were both highly expressed all around the surface of $\mathrm{AR}^{-}$luminal cells compared with adjacent wild-type counterparts (Supplementary Fig. 4e), indicating a disruption of normal cell polarity.

Gene expression profiling analyses of $\mathrm{AR}^{-}$luminal cells. Next, we performed RNA-seq analysis of wild type and $\mathrm{AR}^{-}$luminal cells to compare their gene expression profiles. We isolated wildtype and $\mathrm{AR}^{-}$luminal cells by flow-sorting of $\mathrm{YFP}^{+}$cells from Nkx3.1 CreERT2/+ $^{2}$ R26R-YFP/+ (denoted Lum ${ }^{\text {YFP }}$, control) and Lum ${ }^{\text {YFP,AR - }}$ (experimental) mice 1 month after induction, respectively (Supplementary Fig. 6a). Cytospin analysis of flow-sorted cells showed that $97.6 \%$ of $\mathrm{YFP}^{+}$cells from the experimental mice were $\mathrm{AR}^{-}$, while $99.1 \%$ of $\mathrm{YFP}^{+}$cells from the control mice were $\mathrm{AR}^{+}$(Supplementary Fig. 6b). RNA-seq was performed for eight control and four experimental samples (all were biological replicates). Principal components analysis (PCA) and unsupervised hierarchical clustering analysis demonstrated that the independent samples within each group were consistent and that the control and experimental groups were well separated (Fig. 4a,b). A total of 1,654 genes were upregulated and 1,452 genes were downregulated in $\mathrm{AR}^{-}$luminal cells compared with the wild-type control (Fig. 4c; Supplementary Data 1,2; false discovery rate $($ FDR $)<0.1$, and fold change $>2$ ). As expected, both RNA-seq data and our quantitative real-time PCR results showed that the AR target gene $N k x 3.1$ was downregulated in $\mathrm{AR}^{-}$luminal cells (Fig. 4d; Supplementary Fig. 6c). Notably, both basal and luminal epithelial cell marker genes $(c d h 1, \operatorname{trp63}$, $k r t 8, k r t 14, k r t 18$ ) were upregulated (Fig. 4d; Supplementary Fig. 6c), indicating $\mathrm{AR}^{-}$luminal cells may have molecular features of intermediate cells. Furthermore, genes involved in cell proliferation ( $m k i 67, c c n d 1, c c n d 3, m y c, c d k 4, c d k n 1 a$ ) showed mixed or insignificant expression level changes (Fig. $4 \mathrm{~d}$; Supplementary Fig. 6c), consistent with our finding that $\mathrm{AR}^{-}$ luminal cells at this stage are transitioning away from a hyperproliferative state. DAVID GO analysis ${ }^{40}$ identified 12 enriched molecular pathways in $\mathrm{AR}^{-}$luminal cells (FDR $<0.1$; Supplementary Data 3), with the most notable ones implicated in cell-matrix adhesions, MAPK and TGF- $\beta$ signalling pathways, prostate cancer and cytoskeleton regulation (Fig. 4e). Collectively, these data suggest that $\mathrm{AR}^{-}$luminal cells are primarily altered in cell morphology, and share some molecular signatures with prostate cancer cells.

Luminal cell-autonomous AR is dispensable for regeneration. Classic tissue recombination experiments showed that during organogenesis $\mathrm{AR}^{+}$mesenchyme cells could promote $\mathrm{AR}^{-}$ epithelial cells to grow and generate prostate tissues through paracrine signals ${ }^{4-6}$. However, whether luminal cell-autonomous $\mathrm{AR}$ is required for adult prostate regeneration remains unknown. To test this, we induced $\mathrm{AR}^{-}$luminal cells in 8-week old hormonal-intact Lum ${ }^{\mathrm{YFP}, \mathrm{AR}-}$ mice, and then lineage-traced them in the processes of castration and androgen readministration (Fig. 5a). We found that, after castration, almost all of the $\mathrm{YFP}^{+}$cells $(97.8 \%, n=529 / 541$, three animals analysed) in the regressed prostate were $\mathrm{AR}^{-}$, whereas the unmarked luminal cells showed diffusive AR staining, which would be expected in the absence of androgen (Fig. 5b; Supplementary Fig. 7a). These $\mathrm{YFP}^{+}$cells remained strictly luminal as revealed by $\mathrm{CK} 5$ and $\mathrm{CK} 18$ staining (Fig. 5c; Supplementary Fig. 7a, right panel). Importantly, the percentage of luminal cells that were $\mathrm{YFP}^{+}$or $\mathrm{AR}^{-}$did not change before 
a

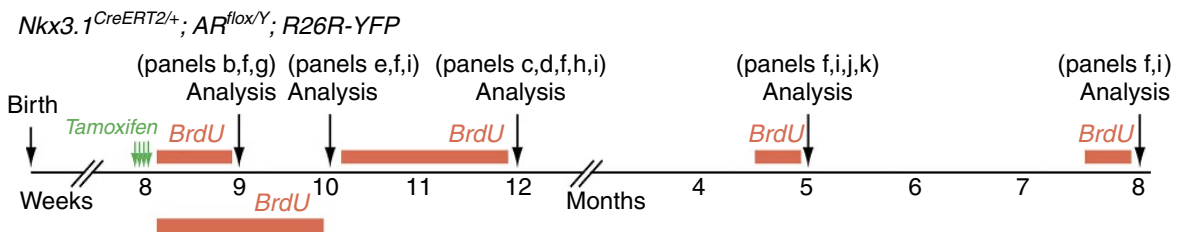

b

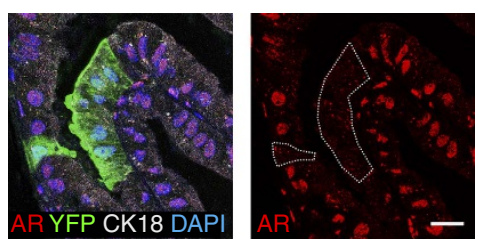

C
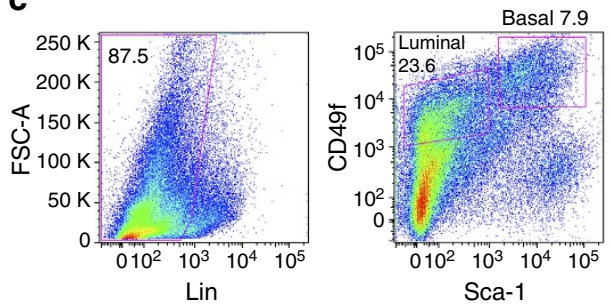

f

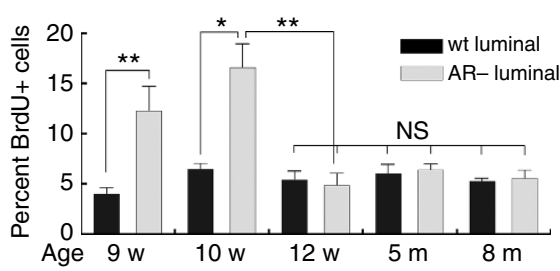

i

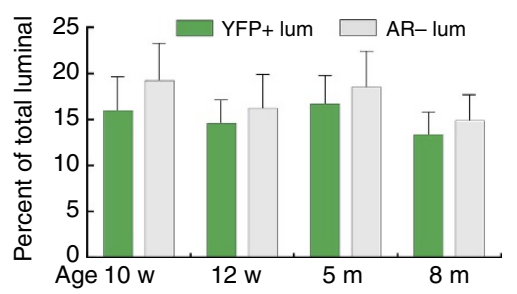

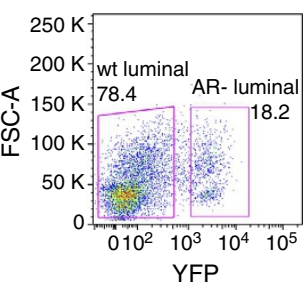

g
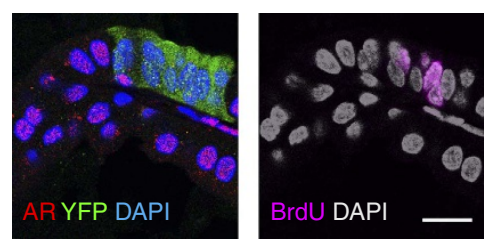

j
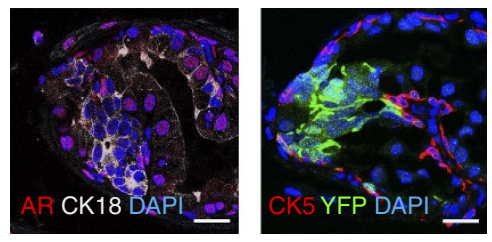

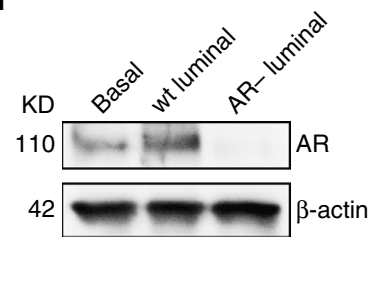

e

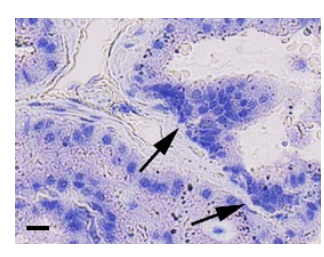

$\mathbf{h}$
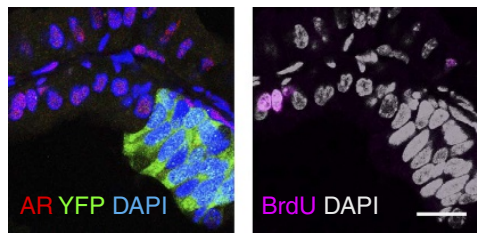

$\mathbf{k}$
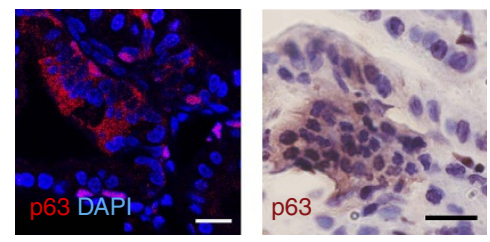

Figure 3 | AR loss in luminal cells induces a transient over-proliferation and alters cell morphology. (a) Lineage-tracing strategy in prostate homeostasis of Lum YFP,AR - mice. (b) Representative IF staining image showing simultaneous deletion of AR and marking by YFP in luminal cells at 1 week post induction. (c) FACS plot showing the gate drawn for obtaining basal, wild-type luminal and AR - luminal cells from induced Lum YFP,AR- mice. (d) Western blot of AR protein from flow-sorted cell populations. (e) H\&E staining showing clusters of compact cells (arrows) at 2 weeks post induction. (f) Quantitation of wild-type and AR - luminal cell proliferation rates using BrdU incorporation assays shown in a, showing a transient (1-2 weeks) over-proliferation in $A R^{-}$luminal cells. ${ }^{\star} P<0.01,{ }^{\star \star} P<0.001$ by $t$-test. (g,h) Representative IF staining images showing many $A R^{-}$luminal cells were $\mathrm{BrdU}^{+}$at 1 week post induction ( $(\mathbf{g})$ and relatively few were $\mathrm{BrdU}^{+}$at 4 weeks post induction (h). (i) Quantitation of the percentage of $\mathrm{YFP}^{+}$or $\mathrm{AR}^{-}$ luminal cells during homeostasis showing no significant difference by $t$-test for different time points since 2 weeks post induction. (j) IF staining of tissues at 3 months post induction showing enhanced CK18 expression (left) and no detectable CK5 expression (right) in AR ${ }^{-} / \mathrm{YFP}^{+}$luminal cell clusters. (k) Cytoplasmic p63 expression was enhanced in AR- luminal cell clusters by both IF (left) and IHC (right) staining using two antibodies. Scale bars correspond to $20 \mu \mathrm{m}$. Error bars correspond to one s.d.

and after castration (Fig. 5f; Supplementary Table 4), indicating that AR-expression levels in luminal cells do not affect the susceptibility/resistance of these cells to androgen deprivation. BrdU incorporation assay was performed for 12 days following testosterone pump implantation (Fig. 5a), and we detected robust proliferation in both $\mathrm{YFP}^{+} \mathrm{AR}^{-}$and $\mathrm{YFP}^{-} \mathrm{AR}^{+}$luminal cells (Fig. 5d; Supplementary Fig. 7b), with rates being similar between the two populations (Fig. 5e; Supplementary Table 4). Ki67 staining performed at 4 days after pump implantation also confirmed this result (Supplementary Fig. 8). Notably, in the fully regenerated prostate, the percentage of $\mathrm{YFP}^{+}$or $\mathrm{AR}^{-}$luminal cells remained unchanged (Fig. 5f; Supplementary Fig. 7d; Supplementary Table 4), and they showed the 'compaction' phenotype (Fig. 5g; Supplementary Fig. 7c). These data demonstrate that cell-autonomous $\mathrm{AR}$ is dispensable for average regressed luminal cells to regenerate. Although the data do not directly prove the case, they strongly support the hypothesis that androgen acts on stromal AR to mediate adult luminal cell regeneration through paracrine signals.

AR is selectively required for CARN stem cell activities. In the regressed prostate, rare castration-resistant $N k x 3.1$-expressing cells (CARNs) were shown to be a type of stem cell that can produce luminal and, to a lesser extent, basal cells during prostate regeneration $^{23}$. To determine the uniqueness of CARNs in prostate regeneration compared with average regressed luminal cells, we next investigated whether their stem cell activities are

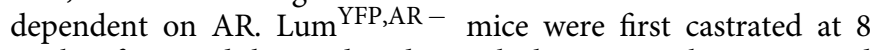
weeks of age and then induced 4 weeks later to mark CARNs and simultaneously delete AR in them (Fig. 6a). Consistent with the study of wild-type CARNs ${ }^{23}$, we found that, in the regressed prostate, $1.0 \%$ of the luminal cells $(n=74 / 7,786$, three animals analysed) were marked by YFP (Fig. 6b). Most of these YFP ${ }^{+}$ 
a

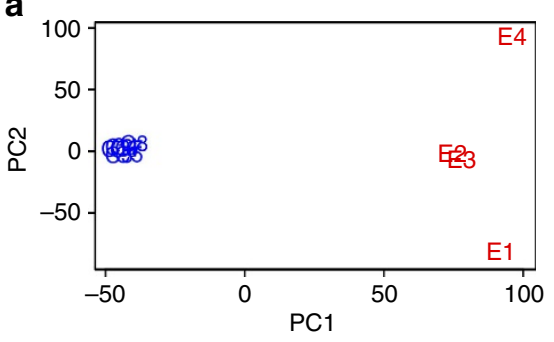

C

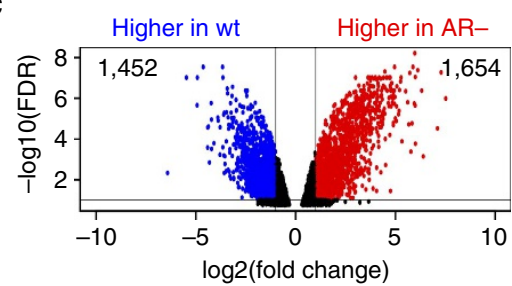

b

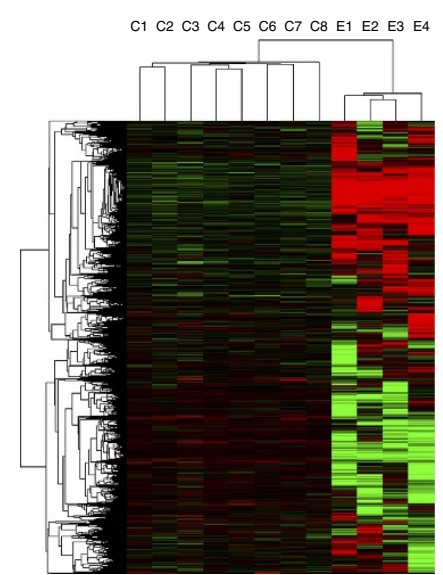

d
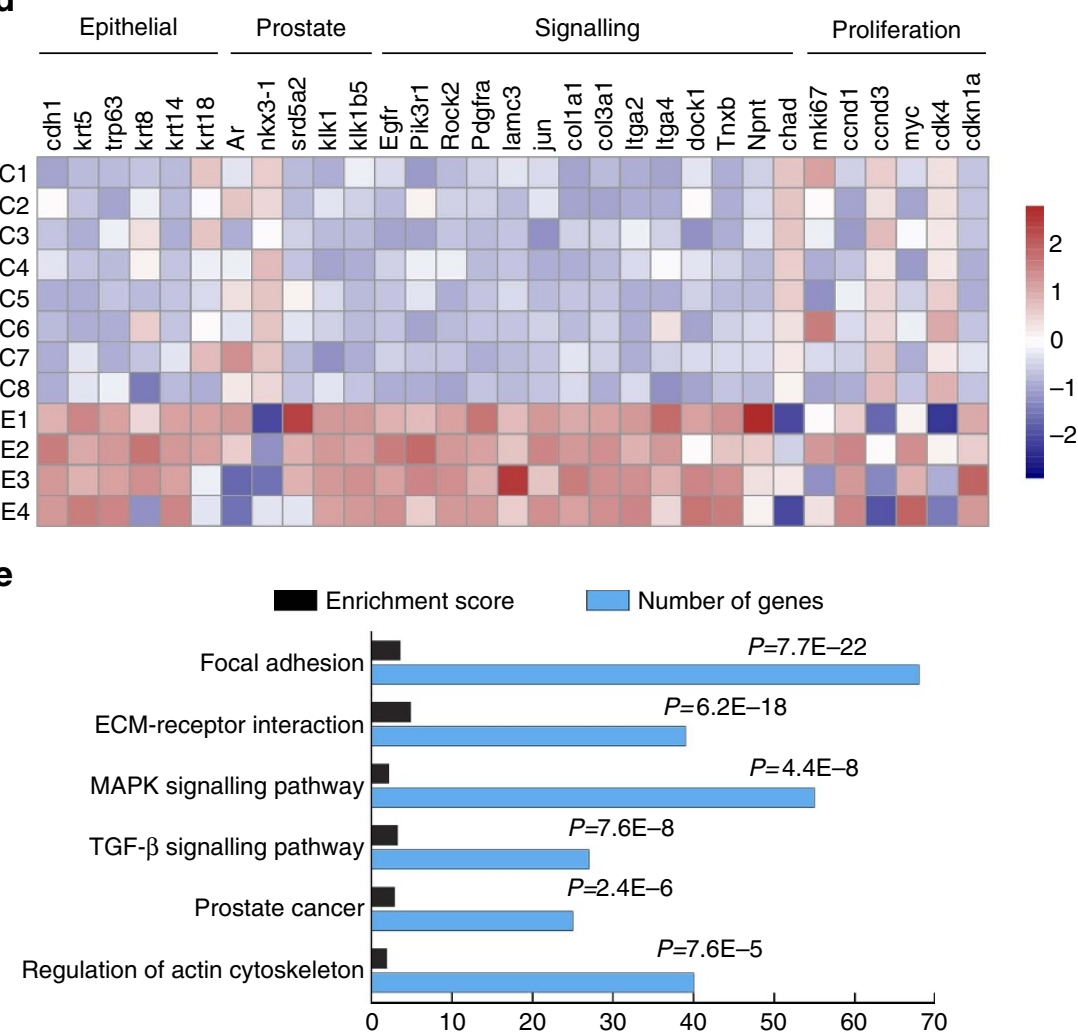

Figure 4 | Gene expression profiling analyses of AR - luminal cells. (a) Scatter-plot of the two main components from a Principal Component Analysis of control (wild-type, blue points) and experimental ( $A R^{-}$, red points) luminal samples based on 9,238 genes after filtering out too lowly or too highly expressed genes, capturing $44.5 \%$ (dimension 1) and $16.6 \%$ (dimension 2) of the data variability. (b) Unsupervised hierarchical clustering analysis showing good separation of control samples (C1-C8) and AR ${ }^{-}$luminal samples (E1-E4). (c) Volcano plot showing 1,654 genes are upregulated and 1,452 genes are downregulated in $A R^{-}$luminal cells (FDR $<0.1$ and fold change $>2$ ). (d) Expression levels of selected genes in different samples showing a general upregulation of epithelial markers and various cell signalling genes in $A R^{-}$luminal cells, and mixed expression pattern changes for proliferation markers. (e) DAVID GO analysis showing the most enriched pathways in $\mathrm{AR}^{-}$luminal cells $(F D R<0.1)$ and the number of genes in each pathway.

cells (82.4\%, $n=61 / 74$, three animals analysed) were $\mathrm{AR}^{-}$ (Fig. 6b), suggesting deletion of AR in CARNs was efficient and did not affect their survival. AR ${ }^{-}$CARNs did not express $N k \times 3.1$ (Supplementary Fig. 9a), indicating cell-autonomous AR directly activates $N k x 3.1$ expression in normal CARNs. Upon completion of prostate regeneration, we detected isolated single $\mathrm{YFP}^{+} \mathrm{AR}^{-}$ cells (Fig. 6c). $\mathrm{YFP}^{+}$cell clusters (defined as $>3$ adjacent cells) in the regenerated prostate were rare, in contrast to results obtained from wild-type CARNs in Lum ${ }^{\text {YFP }}$ mice (Fig. 6d; Supplementary Table 5). Notably, the cells in those rare clusters were $\mathrm{AR}^{+}$ (Fig. 6e), suggesting that they were derived from wild-type CARNs that escaped AR deletion. The same phenotypes were also observed after two rounds of regression-regeneration (Fig. 6f). Surprisingly, the failure of $\mathrm{AR}^{-} \mathrm{CARN}$ to produce cell clusters was not due to a defect in CARN cell proliferation, because we found that $\mathrm{AR}^{+}$and $\mathrm{AR}^{-} \mathrm{CARNs}$ had similar proliferation rates 
a

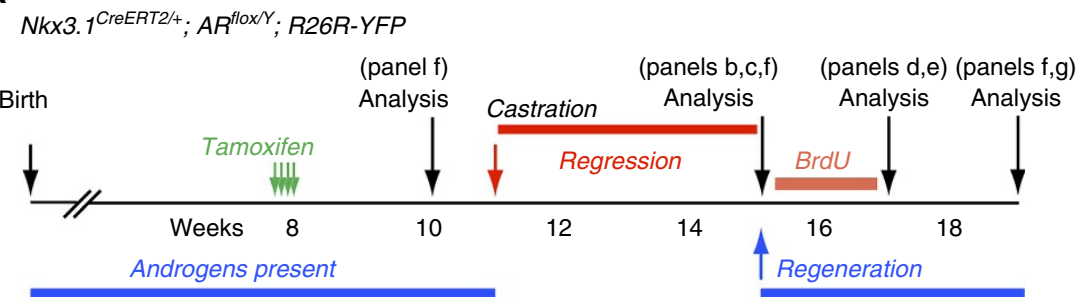

b

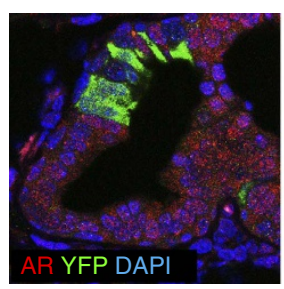

$\mathbf{e}$

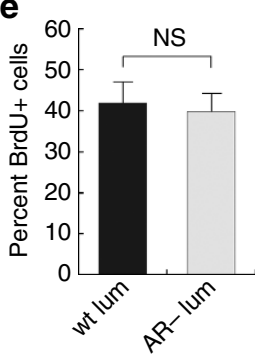

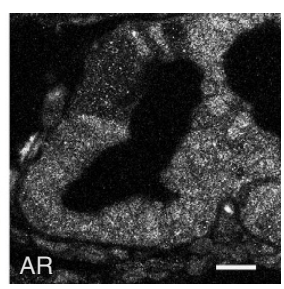

f

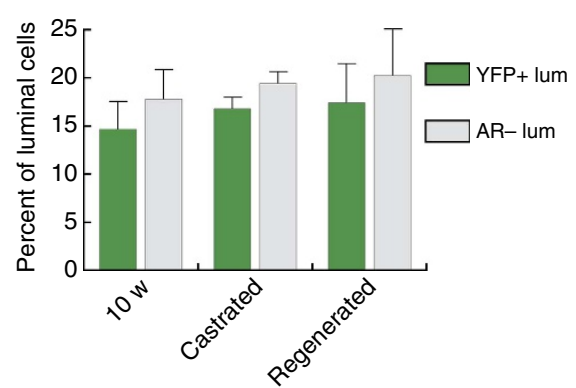

C

d
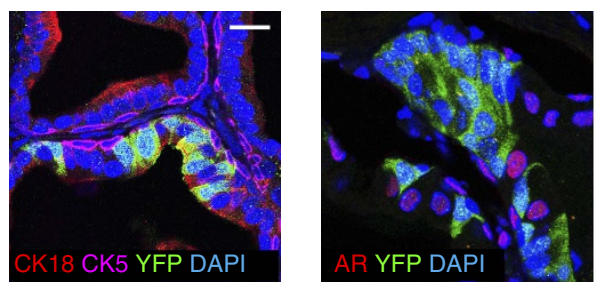

g

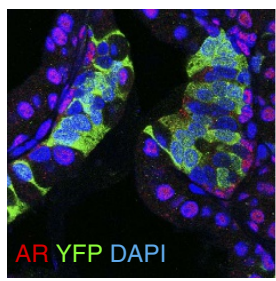

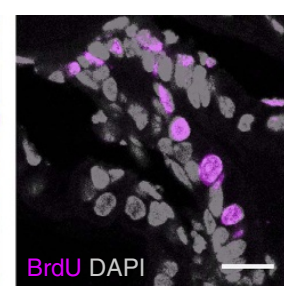

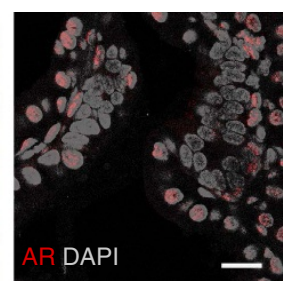

Figure 5 | Cell-autonomous AR is dispensable for luminal cell regeneration. (a) Lineage-tracing strategy for average luminal cells during prostate regeneration in Lum YFP,AR - mice. (b) IF staining showing AR - luminal cells were present in regressed prostate and marked by YFP. (c) CK5, CK18 and YFP triple staining showing marked cells in the regressed prostate remained strictly luminal. (d) Representative image showing high proliferation in both $\mathrm{AR}^{+}$and $\mathrm{AR}^{-}$luminal cells in a BrdU incorporation assay during prostate regeneration. (e) Quantitation of cell proliferation in the BrdU incorporation assay showing no difference by $t$-test between $\mathrm{AR}^{+}$and $\mathrm{AR}^{-}$luminal cells. (f) The percentages of $\mathrm{YFP}^{+}$or $\mathrm{AR}^{-}$luminal cells among total luminal cells during the course of prostate regression-regeneration remained constant by $t$-test. ( $(\mathbf{g}) \mathrm{YFP}^{+} \mathrm{AR}-$ luminal cell clusters were present and had compact cell phenotypes after prostate regeneration. Scale bars correspond to $20 \mu \mathrm{m}$. Error bars correspond to one s.d.

as measured by a BrdU incorporation assay during regeneration (Fig. 6a) as well as Ki67 staining at 3 days post pump implantation (Fig. 6g-i; Supplementary Fig. 9b,c; Supplementary Table 5). Instead, we detected fragmented nuclei and positive-cleaved Caspase 3 signals in adjacent $\mathrm{YFP}^{+}$cells (Fig. 6j), suggesting that the daughter cells of $\mathrm{AR}^{-}$CARNs were apoptotic. These data demonstrate that CARNs selectively require cell-autonomous AR functions to produce viable luminal cells during prostate regeneration, a unique feature that distinguishes them from average luminal cells in the regressed prostate.

To corroborate the above in vivo findings, we also investigated the role of cell-autonomous AR in CARNs using the organoid technique. Lineage-marked CARNs were flowsorted from castrated and induced Lum ${ }^{\mathrm{YFP}, \mathrm{AR}-}$ mice based on YFP fluorescence (Fig. 6a,k). Cytospin analysis of the sorted cells showed that $65.8 \%$ of them had AR deletion $(n=356 / 541$, Fig. 6k). Since CARNs are rare, 867 sorted cells were seeded in a well and organoid culture was performed using a standard serum-free protocol ${ }^{36}$. Ten days later, we found nine organoids that were homogeneously YFP positive (Fig. 61). IF staining revealed that most cells in these organoids were $\mathrm{CK} 18^{+}$luminal (Fig. 6m). Importantly, nuclear AR expression was present in all the cells in eight organoids (Fig. 6m), suggesting they were derived from wild-type CARNs. On the basis of these numbers, we calculated the organoid formation efficiency from wild-type CARNs to be $2.7 \%$, comparable to a previous study using a protocol containing serum ${ }^{35}$. The other organoid contained a mixture of $\mathrm{AR}^{+}$and $\mathrm{AR}^{-}$cells (Fig. 6n), indicating its origin from a doublet composed of one $\mathrm{AR}^{+}$and one $\mathrm{AR}^{-}$CARN cell. In a biological repeat experiment, we again did not observe any pure $\mathrm{AR}^{-}$organoid formation. These data demonstrate that AR is also required for CARN stem cell activities in organoid culture.

Pten loss overrides AR loss in both basal and luminal layers. Having shown the distinct roles of AR in different epithelial cell types in prostate homeostasis, we then explored its cell-typespecific function during cancer initiation. It was discovered that when tumour suppressor gene Pten is deleted, both basal and luminal cells can serve as cells of origin for prostate cancer ${ }^{19,20,27}$. We therefore tested whether AR loss affects tumour initiation from basal and luminal cells under this condition. CK5-CreER ${ }^{T 2}$; $A R^{\text {flox } / Y} ;$ Pten $^{\text {flox/flox }} ;$ R26R-YFP/ + (denoted Bas ${ }^{A R}-$ Pten - $)$ mice and Nkx3.1 $1^{\text {CreERT2/+; }}$ AR flox/Y $;$ Ptenflox/flox; R26R-YFP/+ (denoted Lum $^{\mathrm{AR}-\text { Pten }}{ }^{-}$) mice were induced at 2 month of age and their prostates were analysed at later time points (Supplementary Fig. 10a). One month after induction, the Bas AR - Pten - prostate had an overall normal histology with occasional small foci of hyperplasia. Soon after, Grade II prostatic intraepithelial neoplasia (PIN) lesions began to emerge and were frequent by 3 months after induction. At 6 months after induction, the Bas ${ }^{\mathrm{AR}-\mathrm{Pten}-}$ prostate contained overwhelmingly 
a

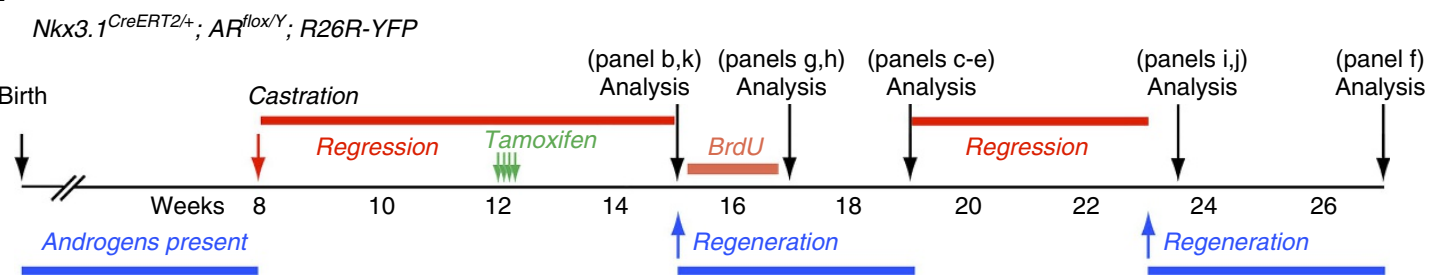

b

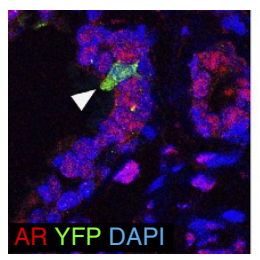

e

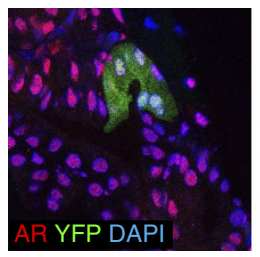

h

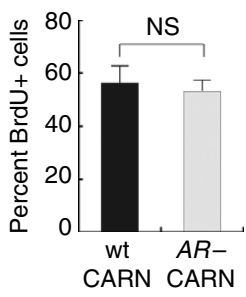

k

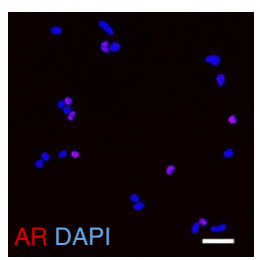

C
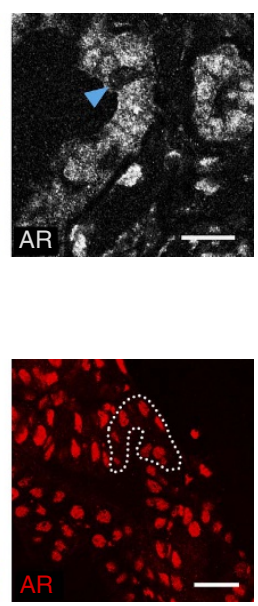

i

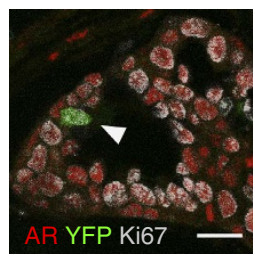

I

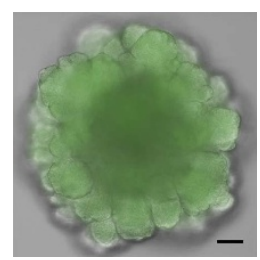

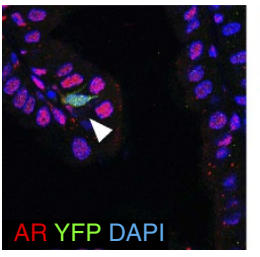

f

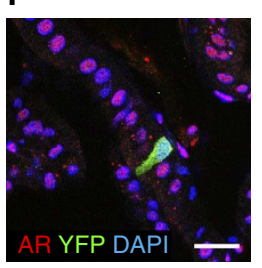

j
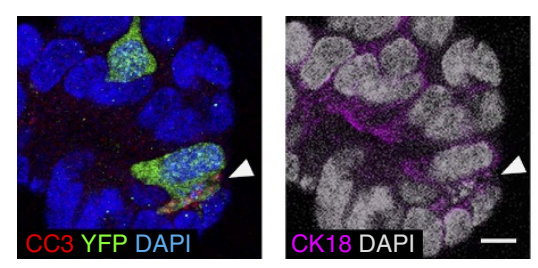

m

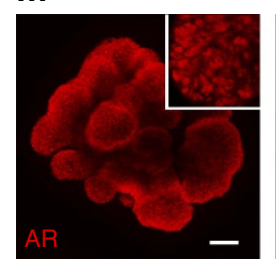

g
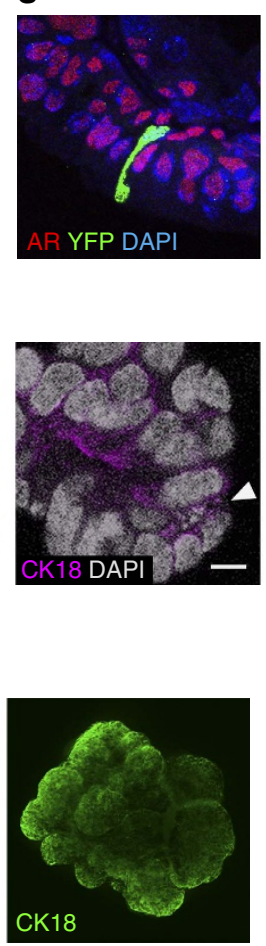

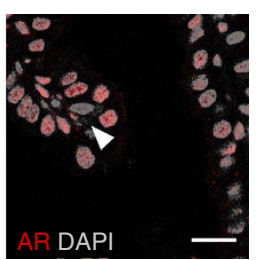

d
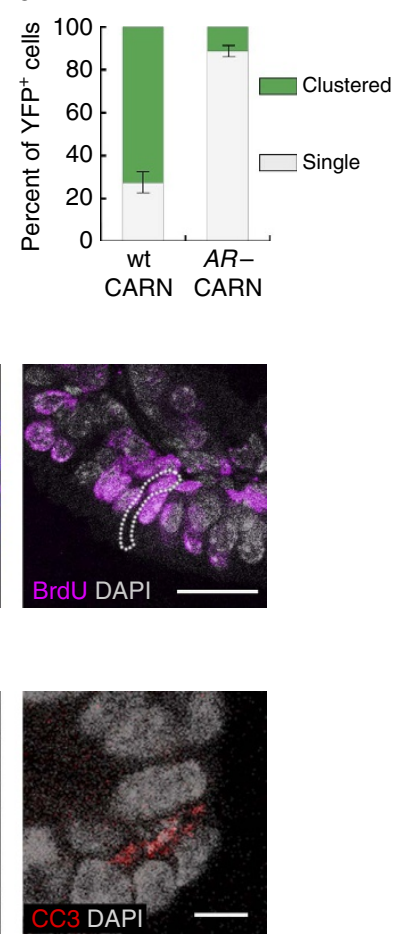

n

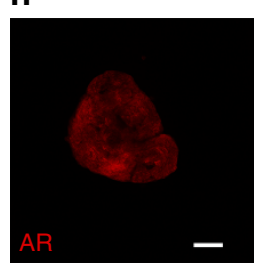

Figure 6 | AR is selectively required for CARN stem cell differentiation. (a) Lineage-tracing strategy for CARNs during serial prostate regressionregeneration in Lum ${ }^{Y F P, A R}$ - mice. (b) Lineage-marked $A R^{-}$CARNs (arrowhead) survived in the regressed prostate after AR deletion. (c) Isolated single $\mathrm{YFP}^{+} \mathrm{AR}^{-}$cells (arrowhead) were present after one round of regeneration. (d) Quantitation of the proportions of clustered and single YFP ${ }^{+}$cells derived from wild-type CARNs (Lum ${ }^{\text {YFP) }}$ ) and $A R^{-}$CARNs (Lum ${ }^{\text {YFP,AR }}{ }^{-}$) after one round of regeneration showing the deficiency of AR ${ }^{-}$CARNs to generate cell clusters by fisher's exact test. (e) Regenerated cells in rare YFP ${ }^{+}$cell clusters in Lum ${ }^{\text {YFP,AR }}-$ mice were $A R^{+}$. (f) Regenerated YFP $^{+}$AR ${ }^{-}$cells remained as single isolated cells after two rounds of regression-regeneration. (g) Representative image showing AR- CARNs were proliferating in a BrdU incorporation assay during prostate regeneration. (h) Quantitation of cell proliferation in the BrdU incorporation assay during prostate regeneration showing no difference between wild-type and AR - CARNs by t-test. (i) Representative image showing an AR - CARN cell (arrowhead) stained positive for Ki67 4 days after androgen re-administration. (j) Cleaved caspase 3 (CC3), YFP and CK18 triple staining showing that the daughter cell (arrowhead) of an $A R^{-}$CARN is apoptotic. (k) IF staining of cytospin preparation of sorted CARN cells from Lum ${ }^{\text {YFP,AR }}-$ mice showing a mixture of $A R^{+}$and $A R^{-} C^{-} A R N s$. (I) Representative white field and YFP overlay image showing morphology of CARN organoid. (m) Representative in situ IF image of an organoid derived from AR ${ }^{+}$CARN. A zoom-in portion shown in inset. (n) IF staining of the only organoid that contained a mixture of $A R^{+}$and $A R^{-}$cells. Scale bars in $\mathbf{b}, \mathbf{c}$, $\mathbf{e - g , i - k}$ correspond to $20 \mu \mathrm{m}$, and in $\mathbf{I}-\mathbf{n}$ to $100 \mu \mathrm{m}$. Error bars correspond to one s.d.

Grade IV PINs with cribriform pattern (Fig. 7a; Supplementary Fig. 10b). In comparison, we found that $\mathrm{Lum}^{\mathrm{AR}-\mathrm{Pten}-}$ tumours progressed much faster than Bas ${ }^{A R}-\mathrm{Pten}-$ tumours, although their eventual high grade PINs were histologically indistinguishable (Fig. 7a; Supplementary Fig. 10b). These findings are highly analogous to the previous findings about basal- and luminal-origin tumours of Pten deletion alone ${ }^{19,20}$. IF staining revealed that most cells in 6-month Bas AR-Ptenand Lum ${ }^{\mathrm{AR}-\text { Pten }-}$ tumours were $\mathrm{AR}^{-}$and phosphor-Akt ${ }^{+}$ (Fig. 7b,c), confirming rapid expansion of $A R^{-}$Pten $^{-}$cells. 
a

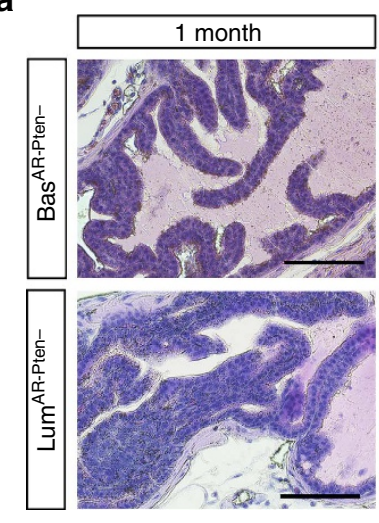

b

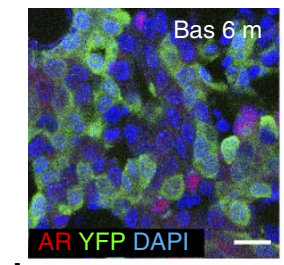

d

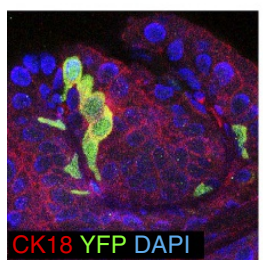

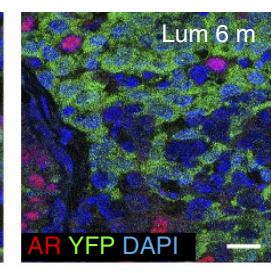

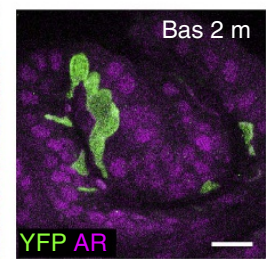

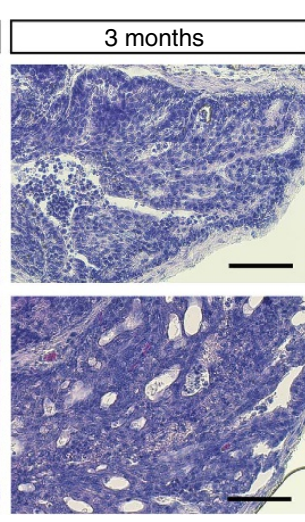
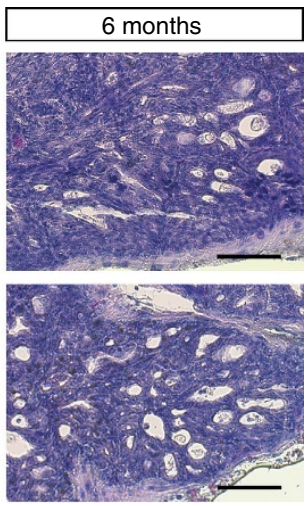

C
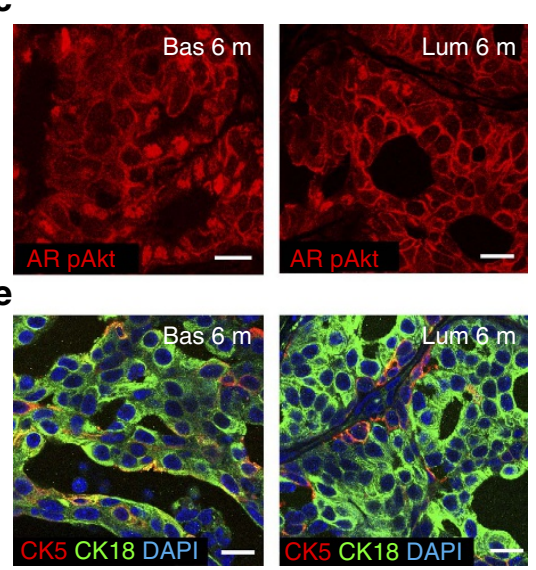

Figure 7 | Pten loss overrides AR-loss effects in both basal and luminal cells during cancer initiation. (a) H\&E staining of Bas ${ }^{A R}-$ Pten - and Lum $^{A R}$ - Pten - AP at 1, 3 and 6 months post induction showing progression to high-grade PIN in both tumours and faster progression in Lum ${ }^{A R}-$ Pten -

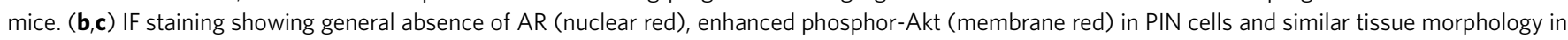
both basal- and luminal-origin tumours at 6 months post induction. (d) Representative image of CK18, YFP and AR triple staining showing AR- Pten basal cells differentiate into luminal cells at 2 months post induction. (e) IF staining showing basal- and luminal-origin tumours at 6 months post induction primarily show luminal cell features with some cells being intermediate ( $\mathrm{CK} 5^{+} \mathrm{CK} 18^{+}$). Scale bars in $\mathbf{a}$ correspond to $100 \mu \mathrm{m}$ and in $\mathbf{b}$-e to $20 \mu \mathrm{m}$.

Interestingly, $A R^{-}$Pten $^{-}$basal cells behaved like Pten $^{-}$basal cells ${ }^{19,20}$ but not $A R^{-}$basal cells, as they readily differentiated into luminal-like cells with enhanced CK18 expression (Fig. 7d). As a result, both Bas ${ }^{A R}$ - Pten - and Lum ${ }^{A R}$ - Pten - tumours were characterized by luminal phenotypes with some cells showing $\mathrm{CK} 5{ }^{+} \mathrm{CK}_{18}{ }^{+}$intermediate features (Fig. 7e). These results suggest that Pten deletion plays a dominant role in the $A R^{-}$Pten $^{-}$double knockout tumours and can override AR-loss effects in both basal and luminal compartments.

Finally, we tested whether AR ${ }^{-}$CARNs can serve as the cell of origin for prostate cancer. Lum ${ }^{A R-P t e n}-$ mice were first castrated, and then induced and re-administered with androgen (Supplementary Fig. 11a). $\mathrm{YFP}^{+} \mathrm{AR}^{-}$tumour cell clusters were readily detected in the regenerated prostate (Supplementary Fig. 11b). The PIN lesions looked similar to previously reported Pten ${ }^{-}$CARN tumours ${ }^{23}$ (Supplementary Fig. 11d), as they expressed high levels of luminal marker CK18 and phosphor-Akt (Supplementary Fig. 11b,c). Therefore, Pten deletion can also override the requirement of $\mathrm{AR}$ in CARNs to transform these stem cells.

\section{Discussion}

A plethora of studies utilizing both tissue recombination and conditional knockout approaches have established the pivotal role of stromal cell AR in instructing epithelial cell proliferation and differentiation in prostate development ${ }^{4-10}$. Here, we demonstrate that AR in adult prostate epithelial cells plays diverse roles in maintaining normal tissue structure, and is crucial for the differentiation capability of adult prostate stem cells in both basal and luminal layers in vivo (Fig. 8).

The present study differs from several previous studies of AR conditional knockout in the prostate. Using Probasin-Cre $(\mathrm{Pb}$-Cre $)$ lines to delete $\mathrm{AR}$ in the developing epithelium, three studies reported increased basal cell proliferation, but conflicting findings regarding luminal cell behaviours. These luminal phenotypes included formation of cell clusters and higher proliferation $^{14}$, little apoptosis in the epithelium, but sloughing of luminal cells into the lumen ${ }^{15}$ and high levels of luminal apoptosis and lower proliferation ${ }^{16}$. The cause for these discrepancies is unknown. In our AR deletion experiments, we did not observe basal cell over-proliferation or luminal cell apoptosis/anoikis. One key distinction is that $\mathrm{Pb}$-Cre lines become active in early postnatal development ${ }^{41}$, whereas the inducible basal and luminal CreER lines were activated by tamoxifen at the adult stage. Therefore, our data reflect homeostatic events in the mature organ, whereas previous studies likely captured developmental consequences of AR loss. As demonstrated previously for prostate basal cells, and stem cells in other organs such as the mammary gland ${ }^{19,42,43}$, cell behaviours including plasticity can be drastically different between 
a
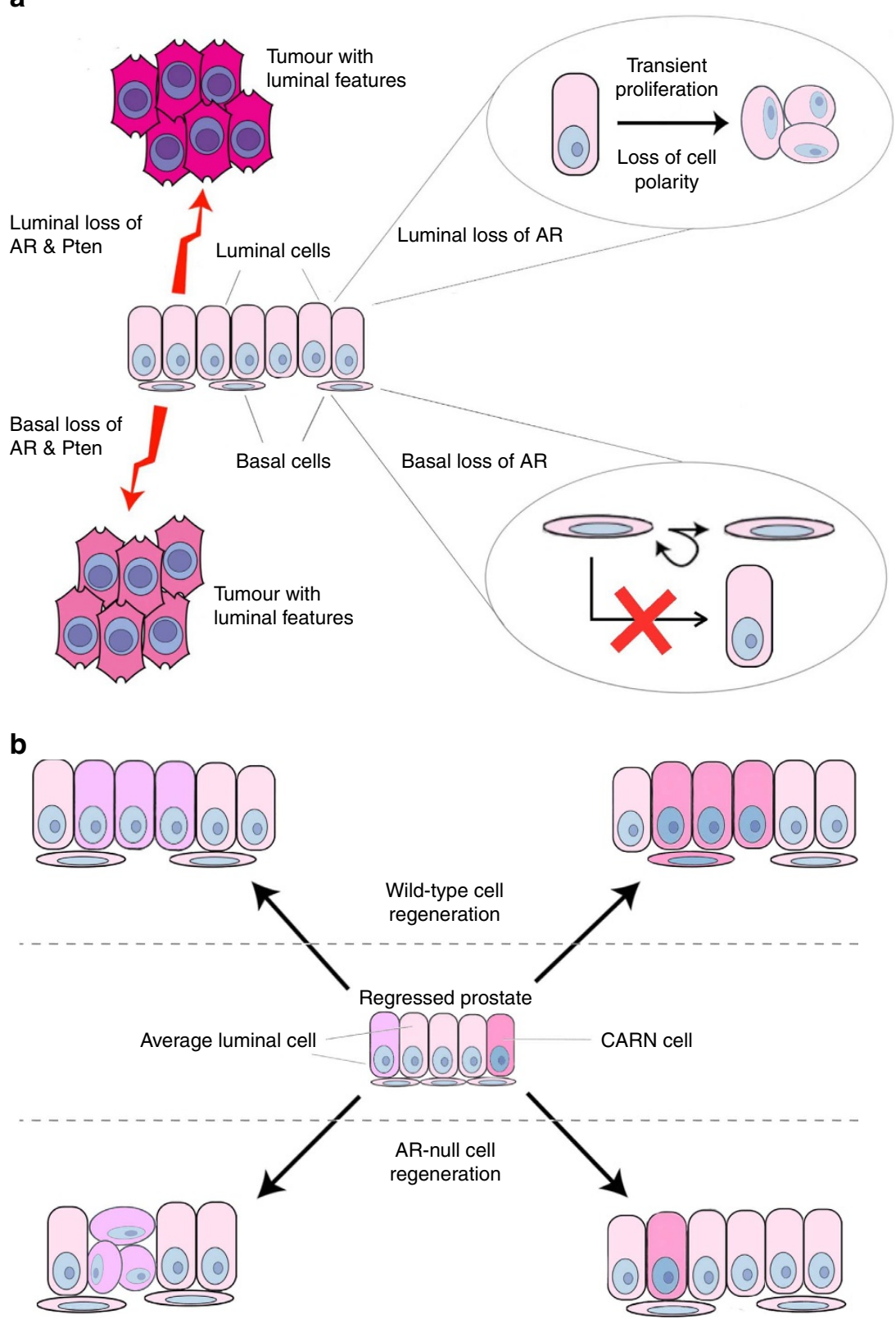

Figure 8 | Model of epithelial AR function in the adult prostate. (a) In adult prostate homeostasis, cell-autonomous AR is essential for maintaining luminal cell morphology and for basal cells to undergo luminal differentiation. It is not required for androgen-mediated cell survival in either compartment. AR loss in luminal cells also induces a transient proliferation, resulting in a cluster of compact cells that share intermediate cell features. Cell-autonomous $A R$ is not required in either basal or luminal cells for Pten-loss-induced cancer initiation. Despite a faster progression rate of luminal-origin tumours with AR Pten double deletion, both basal- and luminal-origin tumours eventually show luminal cell features. (b) In androgen-mediated prostate regeneration, AR is selectively required in luminal stem cell CARNs in order for them to produce viable daughter cells. In contrast, cell-autonomous AR is not required for average luminal cell to proliferate and regenerate upon androgen re-administration, although it ensures the normal morphology and polarity of regenerated luminal cells.

developmental stage and adulthood. During postnatal development, when basal and intermediate cells are actively producing luminal cells ${ }^{18}$, AR loss in luminal cells at this stage might activate a compensatory mechanism to stimulate basal cell proliferation. Alternatively, AR may cell-autonomously repress proliferation of postnatal basal cells, as one study found higher basal cell proliferation when AR was deleted at an early stage by CK5-Cre ${ }^{17}$. Such repression by AR may no longer be needed in the adult basal cells, given that the mature prostate is relatively quiescent and that the plasticity of adult basal cells has become restricted.
Similarly, different timing of Cre activation may also contribute to the reported discrepancies of AR-loss effects on luminal cells. Moreover, characterization of the $\mathrm{Pb}$-Cre4 line has determined that its expression is not only in the epithelium, but also in stromal cells (ref. 41; and our unpublished observations). Therefore, the reported luminal apoptosis phenotypes may alternatively be attributed to $\mathrm{Pb}$-Cre4 leakage and a decrease in stromal AR, since AR conditional knockout in the prostate mesenchyme led to higher epithelial cell apoptosis ${ }^{9,10}$. Our data showing the dispensability of luminal cell-autonomous AR in their survival and regeneration further support the idea that 
androgen regulates luminal cell behaviours primarily in a noncell-autonomous fashion. Instead, luminal cell-autonomous AR is important for maintaining normal luminal cell morphology, possibly through regulating cytoskeleton, cell adhesion and TGF$\beta$ signalling pathways, as indicated by our transcriptome analyses. One potential caveat of the $N k \times 3.1^{C r E R T 2}$ line is that the Cre knock-in disrupts the $N k x 3.1$ gene, which orchestrates a transcriptional regulatory network important for prostate cell fate ${ }^{44}$. However, the Nkx3.1-CreER $R^{T 2 /+}$ mice that we were using were heterozygous and still expressed Nkx3.1 in luminal cells (Supplementary Figs $4 \mathrm{~d}$ and $6 \mathrm{c}$ ), and $N k \times 3.1-/+$ mice were shown to only have very mild phenotypes at old age ${ }^{45}$. Besides, without AR, Nkx3.1 expression is down-regulated anyway. Therefore, we think this technicality is unlikely to affect our conclusions, although the possibility that $n k \times 3.1$ heterozygosity somehow further altered $\mathrm{AR}^{-}$luminal cell properties cannot be totally ruled out.

Interestingly, we find cell-autonomous AR to be essential for stem cell functions in both basal and luminal compartments, although the underlying mechanisms appear to be different. Androgen has been shown to promote basal to luminal cell differentiation in prostate spheres in vitro ${ }^{37,46}$ and in organogenesis ${ }^{47}$. Our data for the first time demonstrate the requirement of cell-autonomous $\mathrm{AR}$ in adult basal cells for their luminal differentiation in vivo. We speculate that rare adult BSCs preserve such a mechanism from postnatal basal cells so that they may step in to generate luminal cells in response to environmental cues such as injury ${ }^{48,49}$. The discovery of a selective AR requirement in luminal CARNs is intriguing. In the regressed prostate, CARNs can regenerate luminal cells (and basal cells to a lesser extent) upon androgen re-administration ${ }^{23}$. Later studies showed that, average regressed luminal cells, which are $N k \times 3.1$-low/negative, also proliferate ${ }^{19}$ and contribute to luminal cell regeneration ${ }^{20,22}$. This raises the question as to whether CARNs are a functionally unique population or rare luminal cells that happen to retain the expression of $N k \times 3.1$. Our data lend support to the former. We show that CARNs, unlike average luminal cells, are sensitive to cell-autonomous AR levels. Importantly, the survival and proliferation of $\mathrm{AR}^{-}$CARNs are not affected, but their differentiated daughter cells are apoptotic. Programed cell death upon cell division was best illustrated in C. elegans neuroblast development, where asymmetric cell division usually generates a smaller daughter cell fated to die, through mechanisms involving EGL-1 and Snail-related proteins $^{50,51}$. It is tempting to speculate that AR loss in CARNs may activate similar mechanisms. Future research will shed light on this, but our organoid culture data and the absence of apoptotic signal in Bas $\mathrm{YFP}, \mathrm{AR}$ - tissues suggest that AR is directly involved in BSC differentiation while its role in CARNs is relatively indirect.

Whether AR is tumour-suppressing or -promoting in prostate cancer is under heated debate $e^{11,12,52}$, and the answer is likely dependent on the specific cell type and progression stage. We found that, despite a transient (1-2 weeks) over-proliferation of $\mathrm{AR}^{-}$luminal cells, their proliferation rates decreased back to normal thereafter, and $\mathrm{AR}^{-}$cell clusters never expanded to colonize the tissue (Fig. 3), suggesting they were not tumorigenic. These data, which were obtained in adult animals, challenge the previous notion that epithelial AR plays a tumour suppressor role during early cancer initiation, since previous experiments were performed at the postnatal stage ${ }^{15,52}$. We acknowledge that in our experiments AR was deleted in $\sim 20 \%$ of all luminal cells. While this number is comparable to luminal marking efficiency in a previous study ${ }^{18}$, it is conceivable that the rest $\mathrm{AR}^{+}$luminal cells can signal to $\mathrm{AR}^{-}$ones and influence their behaviours. Should the AR deletion efficiency be higher, we might observe more prominent PIN-like clustered-cell phenotypes or even un-checked clonal growth. However, we think the later scenario is unlikely, since we did not notice difference with respect to individual cell morphology or proliferation rate between small and large $\mathrm{AR}^{-}$ luminal cell clusters. In future investigations, it will be important to determine whether paracrine signals from $\mathrm{AR}^{+}$luminal cells or perhaps basal and stromal cells caused the over-proliferation of $\mathrm{AR}^{-}$luminal cells to be transient, and whether cell competition exists between $\mathrm{AR}^{-}$and $\mathrm{AR}^{+}$populations. In any case, our luminal AR deletion context may be more physiologically relevant than a situation where deletion occurs ubiquitously (for example, using $\mathrm{Pb}$-Cre), since the appearance of any $\mathrm{AR}^{-}$luminal cells in human prostate should start from a small scale. Finally, our data of AR Pten double knockout in adult basal and luminal cells are consistent with a previous study showing the dispensability of epithelial AR in Pten-null tumour initiation ${ }^{28}$. Mechanistically, Pten loss was found to suppress AR transcriptional output ${ }^{28}$, thereby probably rendering cell-autonomous AR loss to be partially redundant. Indeed, while Pten undergoes copy number loss as a relatively early event in human prostate carcinogenesis ${ }^{53,54}$, mutations of the AR gene have recently been found exclusively in metastatic, castration-resistant human prostate cancer ${ }^{55}$, indicating that AR plays a more prominent role in later-stage cancer progression.

\section{Methods}

Mouse strains and genotyping. The $N k \times 3.1^{\mathrm{CreERT2} /+}$ targeted allele ${ }^{23}$

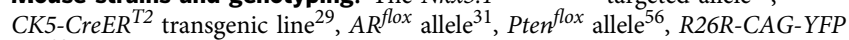
line $^{30}$ and R26R-YFP line ${ }^{57}$ were described previously. Animals were maintained in C57BL/6N background. Genotyping was performed by PCR using tail genomic DNA, with the following primer sequences: $N k \times 3.1$ wild-type allele, $5^{\prime}$-CTCCGCT ACCCTAAGCATCC- $3^{\prime}$ and $5^{\prime}$-GACACTGTCATATTACTTGGACC- $3^{\prime}$; CreER ${ }^{T 2}$ allele, $5^{\prime}$-CAGATGGCGCGGCAACACC- $3^{\prime}$ and $5^{\prime}$-GCGCGGTCTGGCAGTAAA AAC- $3^{\prime} ; A R^{\text {flox }}$ allele, $5^{\prime}$-GTTGATACCTTAACCTCTGC- $3^{\prime}$ and $5^{\prime}$-CTTCAGCG GCTCTTTTGAAG- $3^{\prime}$; Pten ${ }^{\text {flox }}$ allele, $5^{\prime}$-ACTCAAGGCAGGGATGAGC- $3^{\prime}$ and $5^{\prime}$-GTCATCTTCACTTAGCCATTGG-3'; R26R-YFP allele, $5^{\prime}$-GCGAAGAGTTT GTCCTCAACC- $3^{\prime}$ (mutated forward), $5^{\prime}$-GGAGCGGGAGAAATGGATATG- $3^{\prime}$ (wild-type forward) and $5^{\prime}$-AAAGTCGCTCTGAGTTGTTAT- $3^{\prime}$ (wild-type and mutated reverse); R26R-CAG-YFP allele, 5'-AAGGGAGCTGCAGTGGAGTA-3' (wild-type forward), 5'-CCGAAAATCTGTGGGAAGTC-3' (wild-type reverse), $5^{\prime}$-ACATGGTCCTGCTGGAGTTC-3' (mutated forward), 5'-GGCATTAAGCA GCGTATCC-3' (mutated reverse).

Mouse procedures and surgery. For tamoxifen induction, mice were administered $9 \mathrm{mg}$ per $40 \mathrm{~g}$ body weight tamoxifen (Sigma) suspended in corn oil by oral gavage once daily for 4 consecutive days. Castration of adult male mice was performed using standard techniques, with the fully regressed state attained at 4 weeks after castration. For prostate regeneration, testosterone (Sigma) was dissolved at $25 \mathrm{mg} \mathrm{ml}^{-1}$ in $100 \%$ ethanol and diluted in PEG-400 to a final concentration of $7.5 \mathrm{mg} \mathrm{ml}^{-1}$. Testosterone was administered for 4 weeks at a rate of $1.875 \mu \mathrm{gh}^{-1}$ delivered by subcutaneous implantation of mini-osmotic pumps (Alzet), which yields physiological levels of serum testosterone ${ }^{58}$. All animal experiments received approval from the Institutional Animal Care and Use Committee at UCSC. No statistical method was used to predetermine mouse sample size. The mouse experiments were not randomized.

BrdU incorporation assay. BrdU (Sigma) was dissolved in PBS $\left(10 \mathrm{mg} \mathrm{ml}^{-1}\right)$ and administered by intraperitoneal injection twice daily $(0.1 \mathrm{ml}$ per dose) for 7 or 12 consecutive days during homeostasis or regeneration to label proliferating cells.

Tissue collection and flow cytometry. For histological and IF analyses, individual prostate lobes were dissected and fixed in $4 \%$ paraformaldehyde for subsequent cryo-embedding in OCT compound (Sakura), or fixed in $10 \%$ formalin followed by paraffin embedding.

For flow cytometry, prostate tissues were dissected and minced to small clumps, followed by enzymatic dissociation with $0.2 \%$ collagenase I (Invitrogen) in DMEM media with $5 \%$ FBS for $3 \mathrm{~h}$ at $37^{\circ} \mathrm{C}$. Tissues were digested with $0.25 \%$ TrypsinEDTA (StemCell Technologies) for $1 \mathrm{~h}$ at $4{ }^{\circ} \mathrm{C}$, passed through 21- to 26-gauge syringes and filtered through a $40-\mu \mathrm{m}$ cell strainer to obtain single-cell suspensions. Dissociated prostate cells were suspended in Hanks' Balanced Salt Solution Modified/2\% FBS. ROCK inhibitor Y-27632 (StemCell Technologies) was added at $10 \mathrm{uM}$ throughout the whole process to inhibit luminal cell death. Dead cells were excluded by propidium iodide staining and cell sorting was performed on a BD 
FACS Aria II instrument in the Flow Cytometry Shared Facility of UCSC Antibodies used for sorting luminal and basal cells are listed in Supplementary Table 6.

Prostate organoid culture. Flow-sorted $\mathrm{YFP}^{+}$basal or CARN cells were washed with advanced DMEM/F12 (Life Technologies), and resuspended in $10 \mu \mathrm{l}$ advanced DMEM/F12 and $30 \mu \mathrm{l}$ Matrigel per well in the Nunc Lab-Tek II CC2 Chamber Slide System (Fisher). Chamber slide was put upside down in the $37^{\circ} \mathrm{C}$ cell culture incubator for $15 \mathrm{~min}$ to let the matrigel solidify. Mouse prostate organoid culture medium was prepared according a previous protocol ${ }^{36}$. Briefly, the following components were added to advanced DMEM/F12 medium, B27 $(50 \times$ diluted), HEPES $1 \mathrm{M}(100 \times$ diluted), GlutaMAX $(100 \times$ diluted $)$, Penicillin-streptomycin $\left(100 \times\right.$ diluted), N-acetylcysteine $(1.25 \mathrm{mM}), \mathrm{EGF}\left(50 \mathrm{ng} \mathrm{ml}^{-1}\right), \mathrm{A} 83-01(200 \mathrm{nM})$, Noggin $\left(100 \mathrm{ng} \mathrm{ml}^{-1}\right)$, R-spondin $1\left(500 \mathrm{ng} \mathrm{ml}^{-1}\right)$, DHT $(1 \mathrm{nM}), \mathrm{Y}-27632$ dihydrochloride $(10 \mu \mathrm{M})$. Organoid culture medium was prewarmed before adding to the wells. The medium was changed every 2-3 days. Organoids were fixed in $4 \%$ PFA for $20 \mathrm{~min}$ at room temperature, and collected and resuspended in Histogel. Organoids/Histogel mixture was let to solidify at $4{ }^{\circ} \mathrm{C}$ and was embedded in OCT after sucrose treatment. In situ organoid images were taken using the Keyence microscope in the Microscopy Shared Facility of UCSC. Organoid sizes were quantified using ImageJ.

Western blot. Total protein was extracted from flow-sorted cells using T-PER Tissue Protein Extraction Reagent (Fisher), separated by SDS-PAGE and transferred onto PVDF membrane according to standard protocols. Membranes were probed with antibodies directed against AR (sc-815, Santa Cruz Biotechnology, 1:500) and $\beta$-actin (sc-47778, Santa Cruz Biotechnology, 1:500). Signal was visualized with secondary HRP conjugated antibodies and Clarity Western ECL Substrate (Biorad). Full size images are presented in Supplementary Fig. 12.

Quantitative real-time PCR analysis. Quantitative real-time PCR was carried out using Power SYBR Green PCR Master Mix (Life Technology) in the ViiA 7 Real-Time PCR instrument. cDNA samples were diluted 1:100 for all analyses, which were performed in quadruplicate. Expression values were obtained using the $\Delta \Delta C$ T method and normalized to $\beta$-actin (Actb) expression; average values are shown as the mean \pm s.d. Primer sequences are provided in Supplementary Table 7 .

Histology and immunofluorescence staining. H\&E staining was performed using standard protocols on $6 \mu \mathrm{m}$ paraffin sections. Histological assessments were performed using a published classification of mouse PIN lesions ${ }^{59}$. For immunohistochemical staining, $6 \mu \mathrm{m}$ paraffin sections were deparaffinized in xylene, followed by boiling in antigen unmasking solution (Vector Labs). Slides were blocked in 10\% normal goat serum (NGS; Vector Labs), and incubated with primary antibodies diluted in $10 \%$ NGS overnight at $4{ }^{\circ} \mathrm{C}$. Secondary antibodies were obtained from Vectastain ABC kits (Vector Labs) and diluted 1:250. Signal was enhanced using the Vectastain $A B C$ system and visualized with the NovaRed Substrate Kit (Vector Labs). Slides were counterstained with Harris modified haematoxylin (1:4 diluted in $\mathrm{H}_{2} \mathrm{O}$; Fisher Scientific) and mounted with Clearmount (American MasterTech). H\&E and immunohistochemical staining was obtained using a Zeiss Axio Imager in the Microscopy Shared Facility of UCSC.

IF staining was performed using $6 \mu \mathrm{m}$ cryosections $(3 \mu \mathrm{m}$ for staining adjacent sections), which were incubated in $3 \% \mathrm{H}_{2} \mathrm{O}_{2}$ and Antigen Unmasking Solution (Vector Labs) for $15 \mathrm{~min}$. Samples were incubated with $10 \%$ NGS and primary antibodies diluted in $10 \% \mathrm{NGS}$ overnight at $4{ }^{\circ} \mathrm{C}$. Samples were then incubated with secondary antibodies (diluted 1:500 in PBST) labelled with Alexa Fluor 488, 555 or 647 (Invitrogen/Molecular Probes). Detection of Nkx3.1 was enhanced using tyramide amplification (Invitrogen/Molecular Probes) by incubation of slides with HRP-conjugated secondary antibody (1:100 dilution) (Invitrogen/Molecular Probes), followed by incubation with tyramide 555 for 6 min. Slides were mounted with VectaShield mounting medium with DAPI (Vector Labs). IF staining was imaged using a Leica TCS SP5 spectral confocal microscope in the Microscopy Shared Facility of UCSC. All primary antibodies and dilutions used are listed in Supplementary Table 6 .

Cell counting for lineage analyses and statistics. The investigators were blinded to the ID/genotype of the mice before performing cell counting. Cell numbers were counted manually using confocal $\times 40$ and $\times 63$ photomicrographs across tissue sections. Basal cells were identified based on lack of CK18 staining, positivity for CK5 staining, and/or shape of the cells (oval or triangular) and their positions at the basement of the epithelium. Luminal cells were determined based on positive CK18 staining and/or shape of the cells (columnar) and their positions at the apical side of the epithelium. Statistical analyses were performed using a two-sided student's $t$-test, Fisher's exact test, or $\chi^{2}$ test as appropriate. At least three animals for each experiment or genotype were analysed. The variances were similar between the groups that were being statistically compared.

RNA sequencing. Total RNA from FACS-purified luminal cells was isolated using the RNeasy Micro Kit (Qiagen). RNA in each sample was reverse transcribed and amplified into cDNA using the Ovation RNA-Seq System V2 kit (Nugen). The quantity and quality of each sample was measured using an Agilent 2100 Bioanalyzer. Samples were sent to the Columbia Genome Center for library construction and sequencing. The single-end sequencing was performed on the Illumina HiSeq 2000 platform. bc and bcl2fastq (v1.8.4) was used for converting $\mathrm{BCL}$ to fastq format, coupled with adaptor trimming. Sequencing reads were then mapped to mouse genome (mm.9) using TopHat (v2.0.4) by allowing up to four mismatches and ten maximum multiple hits. Expression of genes in the RNA-sec data was measured by calculating reads per kilobase per million mapped reads (FPKM value) using cufflinks (v2.0.2) software with default settings.

Principal components analysis and clustering analysis. PCA was performed on scaled data, where the data value was adjusted by subtracting its mean across all samples and dividing by its s.d., $\mathrm{z}=(\mathrm{x}$-mean $) / \mathrm{s}$.d. For decreasing the effects of potential outliers, the too highly (highest 100 genes according to average FPKM in all samples) or too lowly expressed genes (FPKM $<10$ in all samples) were filtered out. 'pccomp' command in R v3.2.2 was used for PCA analysis. The gene hierarchical clustering was done by using open source clustering software ${ }^{60}$. Here, the Pearson correlation distance was calculated and the average linkage clustering algorithm was chosen.

Gene expression and pathway analyses. Differential expression was estimated using the empirical Bayes methods (limma package ${ }^{61} \mathrm{v} 3.24 .15$ in $\mathrm{R}$ v3.2.2). Fold-change analysis was performed on data regenerated by reverse log transformation. The differentially expressed genes (FDR $<0.1$, and fold change $>2$ ) were extracted and fed to the DAVID website ${ }^{40,62}$ for the enriched pathway analysis.

Data availability. The data that support the findings of this study are available from the corresponding author on request. RNA-seq expression data are deposited in the Gene Expression Omnibus database under GSE76724.

\section{References}

1. Murashima, A., Kishigami, S., Thomson, A. \& Yamada, G. Androgens and mammalian male reproductive tract development. Biochim. Biophys. Acta 1849, 163-170 (2014).

2. Watson, P. A., Arora, V. K. \& Sawyers, C. L. Emerging mechanisms of resistance to androgen receptor inhibitors in prostate cancer. Nat. Rev. Cancer 15, 701-711 (2015).

3. Shen, M. M. \& Abate-Shen, C. Molecular genetics of prostate cancer: new prospects for old challenges. Genes Dev. 24, 1967-2000 (2010).

4. Cunha, G. R. \& Chung, L. W. Stromal-epithelial interactions--I. Induction of prostatic phenotype in urothelium of testicular feminized $(\mathrm{Tfm} / \mathrm{y})$ mice. J. Steroid. Biochem. 14, 1317-1324 (1981).

5. Cunha, G. R. et al. Normal and abnormal development of the male urogenital tract. Role of androgens, mesenchymal-epithelial interactions, and growth factors. J. Androl. 13, 465-475 (1992).

6. Marker, P. C., Donjacour, A. A., Dahiya, R. \& Cunha, G. R. Hormonal, cellular, and molecular control of prostatic development. Dev. Biol. 253, 165-174 (2003).

7. Yu, S. et al. Altered prostate epithelial development and IGF-1 signal in mice lacking the androgen receptor in stromal smooth muscle cells. Prostate 71, 517-524 (2011).

8. Welsh, M. et al. Smooth muscle cell-specific knockout of androgen receptor: a new model for prostatic disease. Endocrinology 152, 3541-3551 (2011).

9. Lai, K. P. et al. Suppressed prostate epithelial development with impaired branching morphogenesis in mice lacking stromal fibromuscular androgen receptor. Mol. Endocrinol. 26, 52-66 (2012).

10. $\mathrm{Yu}, \mathrm{S}$. et al. Altered prostate epithelial development in mice lacking the androgen receptor in stromal fibroblasts. Prostate 72, 437-449 (2012)

11. Singh, M. et al. Stromal androgen receptor in prostate development and cancer Am. J. Pathol. 184, 2598-2607 (2014).

12. Wen, S. et al. Stromal androgen receptor roles in the development of normal prostate, benign prostate hyperplasia, and prostate cancer. Am. J. Pathol. 185, 293-301 (2015).

13. Cunha, G. R. \& Young, P. Inability of Tfm (testicular feminization) epithelial cells to express androgen-dependent seminal vesicle secretory proteins in chimeric tissue recombinants. Endocrinology 128, 3293-3298 (1991).

14. Simanainen, U. et al. Disruption of prostate epithelial androgen receptor impedes prostate lobe-specific growth and function. Endocrinology 148, 2264-2272 (2007).

15. Wu, C. T. et al. Increased prostate cell proliferation and loss of cell differentiation in mice lacking prostate epithelial androgen receptor. Proc. Natl Acad. Sci. USA 104, 12679-12684 (2007).

16. Niu, Y. et al. Increased CK5/CK8-positive intermediate cells with stromal smooth muscle cell atrophy in the mice lacking prostate epithelial androgen receptor. PLoS ONE 6, e20202 (2011). 
17. Lee, S. O. et al. Suppressor role of androgen receptor in proliferation of prostate basal epithelial and progenitor cells. J. Endocrinol. 213, 173-182 (2012).

18. Ousset, M. et al. Multipotent and unipotent progenitors contribute to prostate postnatal development. Nat. Cell Biol. 14, 1131-1138 (2012).

19. Wang, Z. A. et al. Lineage analysis of basal epithelial cells reveals their unexpected plasticity and supports a cell-of-origin model for prostate cancer heterogeneity. Nat. Cell Biol. 15, 274-283 (2013).

20. Choi, N., Zhang, B., Zhang, L., Ittmann, M. \& Xin, L. Adult murine prostate basal and luminal cells are self-sustained lineages that can both serve as targets for prostate cancer initiation. Cancer Cell 21, 253-265 (2012).

21. Tsujimura, A. et al. Proximal location of mouse prostate epithelial stem cells: a model of prostatic homeostasis. J. Cell Biol. 157, 1257-1265 (2002).

22. Liu, J. et al. Regenerated luminal epithelial cells are derived from preexisting luminal epithelial cells in adult mouse prostate. Mol. Endocrinol. 25, 1849-1857 (2011).

23. Wang, X. et al. A luminal epithelial stem cell that is a cell of origin for prostate cancer. Nature 461, 495-500 (2009).

24. Sciavolino, P. J. et al. Tissue-specific expression of murine $N k \times 3.1$ in the male urogenital system. Dev. Dyn. 209, 127-138 (1997).

25. Bieberich, C. J., Fujita, K., He, W. W. \& Jay, G. Prostate-specific and androgen-dependent expression of a novel homeobox gene. J. Biol. Chem. 271, 31779-31782 (1996).

26. Wang, S. et al. Prostate-specific deletion of the murine Pten tumor suppressor gene leads to metastatic prostate cancer. Cancer Cell 4, 209-221 (2003).

27. Lu, T. L. et al. Conditionally ablated Pten in prostate basal cells promotes basal-to-luminal differentiation and causes invasive prostate cancer in mice. Am. J. Pathol. 182, 975-991 (2013).

28. Mulholland, D. J. et al. Cell autonomous role of PTEN in regulating castration-resistant prostate cancer growth. Cancer Cell 19, 792-804 (2011).

29. Rock, J. R. et al. Basal cells as stem cells of the mouse trachea and human airway epithelium. Proc. Natl Acad. Sci. USA 106, 12771-12775 (2009).

30. Madisen, L. et al. A robust and high-throughput Cre reporting and characterization system for the whole mouse brain. Nat. Neurosci. 13, 133-140 (2010).

31. De Gendt, K. et al. A Sertoli cell-selective knockout of the androgen receptor causes spermatogenic arrest in meiosis. Proc. Natl Acad. Sci. USA 101, 1327-1332 (2004).

32. Yeh, S. et al. Generation and characterization of androgen receptor knockout (ARKO) mice: an in vivo model for the study of androgen functions in selective tissues. Proc. Natl Acad. Sci. USA 99, 13498-13503 (2002).

33. Karthaus, W. R. et al. Identification of multipotent luminal progenitor cells in human prostate organoid cultures. Cell 159, 163-175 (2014).

34. Gao, D. et al. Organoid cultures derived from patients with advanced prostate cancer. Cell 159, 176-187 (2014).

35. Chua, C. W. et al. Single luminal epithelial progenitors can generate prostate organoids in culture. Nat. Cell Biol. 16, 951-961 (2014).

36. Drost, J. et al. Organoid culture systems for prostate epithelial and cancer tissue. Nat. Protoc. 11, 347-358 (2016).

37. Lawson, D. A., Xin, L., Lukacs, R. U., Cheng, D. \& Witte, O. N. Isolation and functional characterization of murine prostate stem cells. Proc. Natl Acad. Sci. USA 104, 181-186 (2007).

38. Lukacs, R. U., Goldstein, A. S., Lawson, D. A., Cheng, D. \& Witte, O. N. Isolation, cultivation and characterization of adult murine prostate stem cells. Nat. Protoc. 5, 702-713 (2010).

39. Wang, J. et al. Symmetrical and asymmetrical division analysis provides evidence for a hierarchy of prostate epithelial cell lineages. Nat. Commun. 5 , 4758 (2014).

40. Huang, da, W., Sherman, B. T. \& Lempicki, R. A. Systematic and integrative analysis of large gene lists using DAVID bioinformatics resources. Nat. Protoc. 4, 44-57 (2009).

41. Wu, X. et al. Generation of a prostate epithelial cell-specific Cre transgenic mouse model for tissue-specific gene ablation. Mech. Dev. 101, 61-69 (2001).

42. Wang, Z. A., Toivanen, R., Bergren, S. K., Chambon, P. \& Shen, M. M. Luminal cells are favored as the cell of origin for prostate cancer. Cell Rep. 8, 1339-1346 (2014).

43. Visvader, J. E. \& Stingl, J. Mammary stem cells and the differentiation hierarchy: current status and perspectives. Genes Dev. 28, 1143-1158 (2014).

44. Dutta, A. et al. Identification of an NKX3.1-G9a-UTY transcriptional regulatory network that controls prostate differentiation. Science 352, 1576-1580 (2016).

45. Bhatia-Gaur, R. et al. Roles for $N k \times 3.1$ in prostate development and cancer. Genes Dev. 13, 966-977 (1999).

46. Xin, L., Lukacs, R. U., Lawson, D. A., Cheng, D. \& Witte, O. N. Self-renewal and multilineage differentiation in vitro from murine prostate stem cells. Stem Cells 25, 2760-2769 (2007).

47. Cunha, G. R. et al. The endocrinology and developmental biology of the prostate. Endocr. Rev. 8, 338-362 (1987)
48. Toivanen, R., Mohan, A. \& Shen, M. M. Basal progenitors contribute to repair of the prostate epithelium following induced luminal anoikis. Stem Cell Rep. 6, 660-667 (2016).

49. Kwon, O. J., Zhang, L., Ittmann, M. M. \& Xin, L. Prostatic inflammation enhances basal-to-luminal differentiation and accelerates initiation of prostate cancer with a basal cell origin. Proc. Natl Acad. Sci. USA 111, E592-E600 (2013).

50. Hatzold, J. \& Conradt, B. Control of apoptosis by asymmetric cell division. PLoS Biol. 6, e84 (2008).

51. Potts, M. B. \& Cameron, S. Cell lineage and cell death: Caenorhabditis elegans and cancer research. Nat. Rev. Cancer 11, 50-58 (2011).

52. Niu, Y. et al. Androgen receptor is a tumor suppressor and proliferator in prostate cancer. Proc. Natl Acad. Sci. USA 105, 12182-12187 (2008).

53. Taylor, B. S. et al. Integrative genomic profiling of human prostate cancer. Cancer Cell 18, 11-22 (2010).

54. Baca, S. C. et al. Punctuated evolution of prostate cancer genomes. Cell 153, 666-677 (2013).

55. Robinson, D. et al. Integrative clinical genomics of advanced prostate cancer. Cell 161, 1215-1228 (2015).

56. Lesche, R. et al. Cre/loxP-mediated inactivation of the murine Pten tumor suppressor gene. Genesis 32, 148-149 (2002).

57. Srinivas, S. et al. Cre reporter strains produced by targeted insertion of EYFP and ECFP into the ROSA26 locus. BMC Dev. Biol. 1, 4 (2001).

58. Banach-Petrosky, W. et al. Prolonged exposure to reduced levels of androgen accelerates prostate cancer progression in Nkx3.1; Pten mutant mice. Cancer Res. 67, 9089-9096 (2007).

59. Park, J. H. et al. Prostatic intraepithelial neoplasia in genetically engineered mice. Am. J. Pathol. 161, 727-735 (2002)

60. de Hoon, M. J., Imoto, S., Nolan, J. \& Miyano, S. Open source clustering software. Bioinformatics 20, 1453-1454 (2004).

61. Ritchie, M. E. et al. limma powers differential expression analyses for RNA-sequencing and microarray studies. Nucleic Acids Res. 43, e47 (2015).

62. Huang, da, W., Sherman, B. T. \& Lempicki, R. A. Bioinformatics enrichment tools: paths toward the comprehensive functional analysis of large gene lists. Nucleic Acids Res. 37, 1-13 (2009).

\section{Acknowledgements}

We thank Lindsay Hinck and David States for reading the manuscript, and the microscopy and FACS shared facilities at UCSC for technical support. This work was supported by a post-doctoral fellowship from the CIRM Training Program (Q.X.), UCSC startup fund (Z.A.W.) and by NIH grant GM116872 (Z.A.W.).

\section{Author contributions}

Q.X. and Z.A.W. designed the study. Q.X. performed mouse and molecular experiments with C.H. assistance. Y.L. performed prostate organoid experiments. T.C. performed bioinformatic analyses. Q.X. and Z.A.W. quantified and analysed lineage-tracing data. J.S. contributed to the model figure. All the authors discussed data, and contributed to figures and tables. Z.A.W. wrote the manuscript with input from Q.X., Y.L. and T.C.

\section{Additional information}

Supplementary Information accompanies this paper at http://www.nature.com/ naturecommunications

Competing financial interests: The authors declare no competing financial interests.

Reprints and permission information is available online at http://npg.nature.com/ reprintsandpermissions/

How to cite this article: Xie, Q. et al. Dissecting cell-type-specific roles of androgen receptor in prostate homeostasis and regeneration through lineage tracing. Nat. Commun. 8, 14284 doi: 10.1038/ncomms14284 (2017).

Publisher's note: Springer Nature remains neutral with regard to jurisdictional claims in published maps and institutional affiliations.

This work is licensed under a Creative Commons Attribution 4.0 International License. The images or other third party material in this article are included in the article's Creative Commons license, unless indicated otherwise in the credit line; if the material is not included under the Creative Commons license, users will need to obtain permission from the license holder to reproduce the material. To view a copy of this license, visit http://creativecommons.org/licenses/by/4.0/

(C) The Author(s) 2017 